\title{
Misleading results from low-resolution spectroscopy: From galaxy interstellar medium chemistry to cosmic star formation density ${ }^{\star}$
}

\author{
Y. C. Liang ${ }^{1}$, F. Hammer ${ }^{1}$, H. Flores ${ }^{1}$, N. Gruel ${ }^{2,1}$, and F. Assémat ${ }^{1}$ \\ 1 GEPI, Observatoire de Paris-Meudon, 92195 Meudon, France \\ e-mail: [yanchun.liang, francois.hammer]@obspm.fr \\ 2 Department of Astronomy, University of Florida, 216 Bryant Space Science Center, PO Box 112055, \\ Gainesville, FL 32611-2055, USA
}

Received 21 February 2003 / Accepted 19 December 2003

\begin{abstract}
Low resolution spectroscopy $(R=150)$ from the Canada-France-Redshift Survey (CFRS) revealed intriguing properties for low redshift galaxies $(z \leq 0.3)$ : nearly half of their spectra show prominent $\mathrm{H} \alpha$ emission lines, but no $\mathrm{H} \beta$ emission line and barely detected [O II] $\lambda 3727$, and [O III] $\lambda 5007$ lines. We call these objects "CFRS $\mathrm{H} \alpha$-single" galaxies and have reobserved a subsample of them at higher spectral resolution, associated with a subsample of more normal emission line galaxies. Good S/N spectroscopy at the VLT and the CFHT, with moderate spectral resolution $(R>600)$, have allowed us to perform a full diagnostic of their interstellar medium chemistry. "CFRS $\mathrm{H} \alpha$-single" galaxies and most of the star forming spirals have high extinctions $\left(A_{\mathrm{V}}>2\right)$, high stellar masses and over-solar oxygen abundances.

From the present study, we find it hazardous to derive the detailed properties of galaxies (gas chemical abundances, interstellar extinction, stellar population, star formation rates and history) using spectra with resolutions below 600. One major drawback is indeed the estimated extinction which requires a proper analysis of the underlying Balmer absorption lines. We find that, with low resolution spectroscopy, star formation rates (SFRs) can be either underestimated or overestimated by factors reaching 10 (average 3.1), even if one accounts for ad hoc extinction corrections. These effects are prominent for a large fraction of evolved massive galaxies especially those experiencing successive bursts (A and F stars dominating their absorption spectra). Further estimates of the cosmic star formation density at all redshifts mandatorily requires moderate resolution spectroscopy to avoid severe and uncontrolled biases.
\end{abstract}

Key words. galaxies: abundances - galaxies: photometry - galaxies: evolution - galaxies: spiral - galaxies: starburst

\section{Introduction}

Star formation history is a fundamental quantity to study the populations and evolution of galaxies. Hot, massive, short-lived OB stars emit ultraviolet (UV) photons which ionize the surrounding gas to form an $\mathrm{H}$ II region, where the recombinations produce spectral emission lines. Among the Balmer lines, $\mathrm{H} \alpha$ is the most directly proportional to the ionizing UV stellar spectra at $\lambda<912 \AA$ (Osterbrock 1989), and the weaker Balmer lines are much more affected by stellar absorption and reddening. The other commonly observed metallic optical lines

Send offprint requests to: Y. C. Liang, e-mail: yanchun. liang@obspm. fr

* Based on observations collected with the ESO Very Large Telescope at the Paranal Observatory (under programmes 66.A0599(A) and 67.A-0218(A)) and with the Canada-France-Hawaii Telescope (under programmes 96IIF25, 98IIF16, 98IF65A and 98IIC14), which is operated by CNRS of France, NRC of Canada, and the University of Hawaii. such as [N II] $\lambda \lambda 6548,6583$, [S II] $\lambda \lambda 6716,6731$, [O II] $\lambda 3727$, and [O III] $\lambda \lambda 4958,5007$ depend strongly on the metal fraction present in the gas. Their ionizing potential is higher than the Balmer lines and thus depends on the hardness of the ionizing stellar spectra. These metal lines are indirect tracers of recent star formation, and they characterize the gas chemistry which is linked to the star formation history in individual galaxies. Hence, $\mathrm{H} \alpha$ luminosity density is a strong tool to estimate the cosmic star formation density (CSFD).

The optical $\mathrm{H} \alpha$ line has been used to estimate the star formation (SF) density in the nearby universe. Tresse \& Maddox (1998, hereafter TM98) have calculated the H $\alpha$ luminosity density at $z \sim 0.2$ on the basis of $z<0.3$ CFRS galaxies and have obtained $10^{39.44 \pm 0.04} \mathrm{ergs} \mathrm{s}^{-1} \mathrm{Mpc}^{-1}$. More recent works (Pascual et al. 2001; Fujita et al. 2003), based on deep imaging narrow band data, provided respectively 1.6 and 1.9 times higher than TM98 estimated value. Fujita et al. (2003) argued that their estimates correspond to a redshift (0.24) slightly higher than that sampled by TM98, and also that their data 
revealed a steeper slope of the faint end of the $\mathrm{H} \alpha$ luminosity function.

The above works were based on narrow band filter data and spectroscopy of very low resolving powers ( $R$ from 65 to 150$)$ and they made crude assumptions about the extinction coefficient to be applied on the $\mathrm{H} \alpha$ luminosity. Indeed, at low resolving power, the underlying Balmer absorption, related to intermediate age stellar populations, can severely affect the $\mathrm{H} \alpha / \mathrm{H} \beta$ ratio used to estimate the extinction coefficient.

In this paper, our aim is to address two questions: (1) can one estimate the global properties (gas chemical abundances, extinction and SFRs) of individual galaxies on the basis of low resolution spectroscopy? (2) How robust are SF density estimates based on low resolution spectroscopy or narrow band filters imagery? To tackle these issues we have gathered a small, but representative sample of low $-z$ galaxies and have systematically compared their properties derived from different spectral resolutions, from very low to moderate ones. Low resolution spectroscopy (about $40 \AA$ ) was provided by the CFRS spectra, and moderate resolution spectra have been obtained by using the European Southern Observatory (ESO) Very Large Telescope (VLT) and the Canada-France-Hawaii Telescope (CFHT): VLT/FORS and CFHT/MOS (5 A or $12 \AA$ for VLT, $12 \AA$ for CFHT)

The paper is organized as follows. In Sect. 2, we describe the sample selection. The observations, data reduction and flux measurements are described in Sect. 3. Extinction properties are discussed in Sect. 4, allowing to present diagnostic diagrams and the estimated gas abundances in Sect. 5. The derived SFRs of these galaxies were given in Sect. 6, which includes a discussion on the requirements needed for proper estimates. For reasons of consistency with the former analyses, the adopted cosmological constants are $H_{0}=50 \mathrm{~km} \mathrm{~s}^{-1} \mathrm{Mpc}^{-1}$ and $q_{0}=0.5$ throughout this paper.

\section{CFRS low redshift galaxies: The sample selection}

CFRS has produced a unique sample of 591 field galaxies with $I_{\mathrm{AB}}<22.5$ in the range $0<z<1.3$ with a median $\langle z\rangle \sim 0.56$ (Lilly et al. 1995a,b; Le Fèvre et al. 1995; Hammer et al. 1995; Crampton et al. 1995), which is a good sample to study star formation history, stellar population and evolution of galaxies.

There are 138 CFRS galaxies with $z \leq 0.3$ showing both $\mathrm{H} \alpha$ and $\mathrm{H} \beta$ lines in the rest-frame optical wavelength range. 21 of these galaxies exhibit both $\mathrm{H} \alpha$ and $\mathrm{H} \beta$ in absorption, and 117 exhibit $\mathrm{H} \alpha$ emission lines (see Fig. 1). Among the 117 low- $z$ H $\alpha$ emission galaxies, 57 ( 49\%) exhibit nonpositive equivalent width of $\mathrm{H} \beta, E W(\mathrm{H} \beta) \leq 0$, (zero in 43 and negative in 14 galaxies), and generally exhibit no [O III] $\lambda 5007$, [O II] $\lambda 3727$ emission lines (Hammer et al. 1997; Hammer $\&$ Flores 2001; Tresse et al. 1996). We call these galaxies "CFRS $\mathrm{H} \alpha$-single" galaxies. 53 of the $\mathrm{H} \alpha$-emission line galaxies show $E W(\mathrm{H} \beta)>0$, which are called as "CFRS normal emission line" galaxies in this study. The other 7 are without available information about $\mathrm{H} \beta$ because of the weak quality of their spectra near to $4861 \AA$ wavelength (Tresse et al. 1996; Hammer et al. 1997).

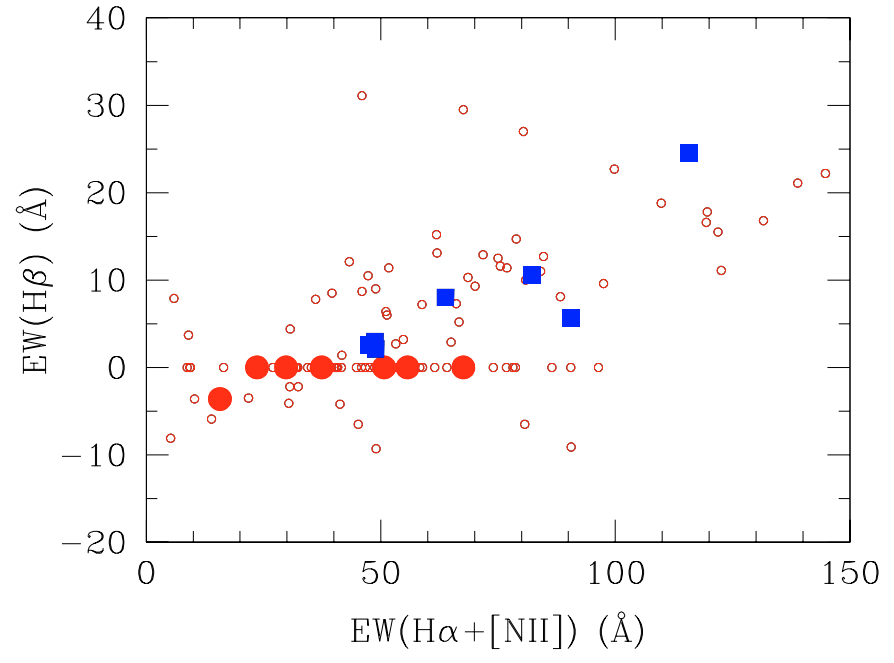

Fig. 1. $E W(\mathrm{H} \beta)$ against $E W(\mathrm{H} \alpha+[\mathrm{N} I \mathrm{II}])$ for the $117 \mathrm{H} \alpha$ emission CFRS galaxies $(z \leq 0.3)$ (open circles) at rest-frame. Five galaxies are outside the diagram, having higher $E W\left(\mathrm{H} \alpha+\left[\mathrm{N}_{\mathrm{II}}\right]\right)$ than $150 \AA$, including two sample galaxies in this study, CFRS22.0474 with (370, $42)$ and CFRS14.1103 with $(2940,192)$. The large filled circles represent the "CFRS $\mathrm{H} \alpha$-single" galaxies. The filled squares represent the "CFRS normal emission line" galaxies.

Why do almost half of the CFRS low- $z$ sample galaxies exhibit "H $\alpha$-single" spectra? Could it be due to large extinctions? Is it related to the low spectral resolution in the CFRS? Or could it be due to the "fact" that they are "peculiar" objects? What is the difference or relation between the "H $\alpha$-single" galaxies and other "normal emission line" galaxies?

Seven "CFRS H $\alpha$-single" galaxies are selected to understand their detailed properties (the filled circles in Fig. 1). Another nine "CFRS normal emission line" galaxies (showing both $\mathrm{H} \alpha$ and $\mathrm{H} \beta$ in emission) are selected for comparison (the squares in Fig. 1). They represent the two group galaxies mentioned above as demonstrated in Fig. 1, although all of them were observed during observational runs targetting different goals (higher redshift galaxies) by completing MOS or FORS masks.

The properties of the 16 sample galaxies are studied by using moderately high resolution and high $\mathrm{S} / \mathrm{N}$ spectra from the VLT and the CFHT. Their images were obtained by the Hubble Space Telescope (HST) WFPC2 with filter F814W in 1994 and the CFHT/FOCAM in 1991. The basic data for these galaxies are given in Table 1 . The columns are CFRS name, redshift, $I_{\mathrm{AB}}$ magnitude, absolute $B$ magnitude, $K_{\mathrm{AB}}$ and absolute $K$ magnitudes ("9999" was marked for the absent values), and the telescopes used for spectral and imaging observations. All the magnitudes are in the AB systems.

\section{Observations, data reduction and flux measurements}

Spectrophotometric observations for four sample galaxies were obtained during one night (for $3^{\mathrm{h}}$ field) with the ESO $8 \mathrm{~m}$ VLT/FORS2 instrument with the R600 and I600 grisms $(R=5 \AA)$ and covering the wavelength range from 5500 to $9200 \AA$. The slit width was $1.2^{\prime \prime}$, and the 
Table 1. Basic data of the sample galaxies, the top seven are the "CFRS $\mathrm{H} \alpha$-single" galaxies, the bottom nine are the "CFRS normal emission line" galaxies. "SPE" means "spectroscopy" and "IMA" means "imagery".

\begin{tabular}{cccccccc}
\hline \hline Objects & $z$ & $I_{\mathrm{AB}}$ & $M_{B}$ & $K_{\mathrm{AB}}$ & $M_{K}$ & SPE & IMA \\
\hline 03.0364 & 0.2511 & 19.05 & -20.79 & 17.89 & -22.63 & CFHT & HST \\
03.0365 & 0.2183 & 19.19 & -20.09 & 17.91 & -22.20 & CFHT & HST \\
03.0578 & 0.2192 & 20.79 & -19.16 & 20.07 & -20.04 & VLT600 & HST \\
03.0641 & 0.2613 & 20.03 & -19.62 & 9999 & 9999 & VLT600 & CFHT \\
03.0711 & 0.2615 & 21.04 & -19.03 & 19.76 & -20.92 & VLT600 & HST \\
03.1014 & 0.1961 & 18.42 & -20.87 & 17.27 & -23.10 & CFHT & HST \\
22.0717 & 0.2791 & 19.60 & -20.24 & 17.92 & -23.04 & VLT300 & CFHT \\
\hline 03.0003 & 0.2187 & 22.49 & -16.60 & 21.81 & -18.18 & CFHT & CFHT \\
03.0149 & 0.2510 & 20.74 & -19.42 & 19.70 & -20.82 & VLT600 & HST \\
03.0160 & 0.2184 & 21.83 & -17.71 & 9999 & 9999 & CFHT & CFHT \\
03.1299 & 0.1752 & 18.59 & -20.47 & 9999 & 9999 & CFHT & HST \\
03.1311 & 0.1755 & 19.56 & -19.40 & 18.05 & -22.23 & CFHT & HST \\
14.1103 & 0.2080 & 22.33 & -17.69 & 9999 & 9999 & CFHT & CFHT \\
14.1117 & 0.1919 & 20.79 & -18.98 & 9999 & 9999 & CFHT & CFHT \\
22.0474 & 0.2801 & 21.74 & -18.66 & 21.12 & -19.55 & VLT300 & CFHT \\
22.1084 & 0.2930 & 20.29 & -20.22 & 19.22 & -21.70 & CFHT & CFHT \\
\hline
\end{tabular}

slit length $10^{\prime \prime}$. The objects CFRS22.0474 and CFRS22.0717 were observed in another night (for $22^{\mathrm{h}}$ field) using the VLT/FORS2 instruments with the R300 grism $(R=12 \AA)$ and covering the wavelength range from 5800 to $9500 \AA$. The slit width was $1.0^{\prime \prime}$, and the slit length $10^{\prime \prime}$. CFHT data were obtained in different runs from 1996 to 1999 , using the standard MOS setup with the R300 grism and a 1.5" slit. These ensure the coverage from $\mathrm{H} \beta$ to [S II] lines in the rest-frame spectrum.

The spectra were extracted and wavelength-calibrated using $\mathrm{IRAF}^{1}$ packages. Flux calibration was done using $15 \mathrm{~min}$ exposures of different photometric standard stars. To ensure the reliability of the data, all spectrum extractions, as well as the lines measurements, were performed by using the SPLOT program.

The rest-frame spectra of the 16 sample galaxies are given in Figs. 2a-o and Figs. 3a,b. The continua have been convolved except at the locations of the emission lines (e.g. $\mathrm{H} \beta ;$ [O III] $\lambda \lambda 4958,5007 ;$ [N II] $\lambda \lambda 6548,6583 ; \mathrm{H} \alpha$ and [S II] $\lambda \lambda 6716,6731)$ using the procedure developed by our group (Hammer et al. 2001; Gruel 2002; Gruel et al. 2003). For the VLT600 spectra, the adopted convolution factors are 7 pixels and then 15 pixels; for the VLT300 and CFHT spectra, the convolution factors are 7 and 7 pixels (Hammer et al. 2001; Gruel et al. 2003). Pairs of vertical dashed lines delimit the regions where strong sky emission lines (e.g. [OI] 5577, 6300, $6364 \AA$ and $\mathrm{OH} 6834,6871,7716,7751,7794,7851$, 7914, 7993, 8289, 8298, 8344, $8505 \AA$ ) and absorption lines $\left(\mathrm{O}_{2} 6877,7606\right.$ and $\left.7640 \AA\right)$ are located. Figures $2 \mathrm{a}^{\prime}-\mathrm{o}^{\prime}$ and

${ }^{1}$ IRAF is distributed by the National Optical Astronomical Observatories, which are operated by the Association of Universities for Research in Astronomy, Inc., under cooperative agreement with the National Science Foundation.
Figs. 3c,d give the corresponding CFRS low-resolution spectra of the galaxies. The comparison between the moderateresolution spectra and the CFRS spectra show that the emission lines are strongly hidden or diluted in the low resolution observations. And the higher resolution make it possible to separate the $[\mathrm{N} \mathrm{II}] \lambda \lambda 6548,6583$ emission lines from the $\mathrm{H} \alpha$ emission.

The fluxes of emission lines have been measured using the SPLOT package. The stellar absorption under the Balmer lines are estimated from the synthesized stellar spectra obtained using the stellar spectra of Jacoby et al. (1984). The corresponding error budget has been deduced using three independent methods: the first one is estimated by trying several combinations of the stellar templates for the stellar absorption; the second one is from measurement, which is estimated according to the independent measurements performed by Liang, Flores and Hammer; the third one is Poisson noise from both sky and objects. The flux measurements of the emission lines from the VLT and the CFHT spectra and their errors are given in Table 2. The fluxes of [O II] $\lambda 3727$ are estimated from the original CFRS spectra with large error bars due to the absence of this line in the rest-frame wavelength ranges of the moderate resolution spectra.

\section{Balmer decrement and extinction}

The major factor that affects measurements of the true emission fluxes is interstellar extinction. If galaxies are observed at high galactic latitudes, the extinction due to our own Galaxy is negligible ( $\sim 0.05 \mathrm{mag})$, hence most extinction is intrinsic to the observed galaxy. Extinction arising along the line of sight to a target galaxy makes the observed ratio of the flux of two emission lines differ from their ratio as emitted in the galaxy. 

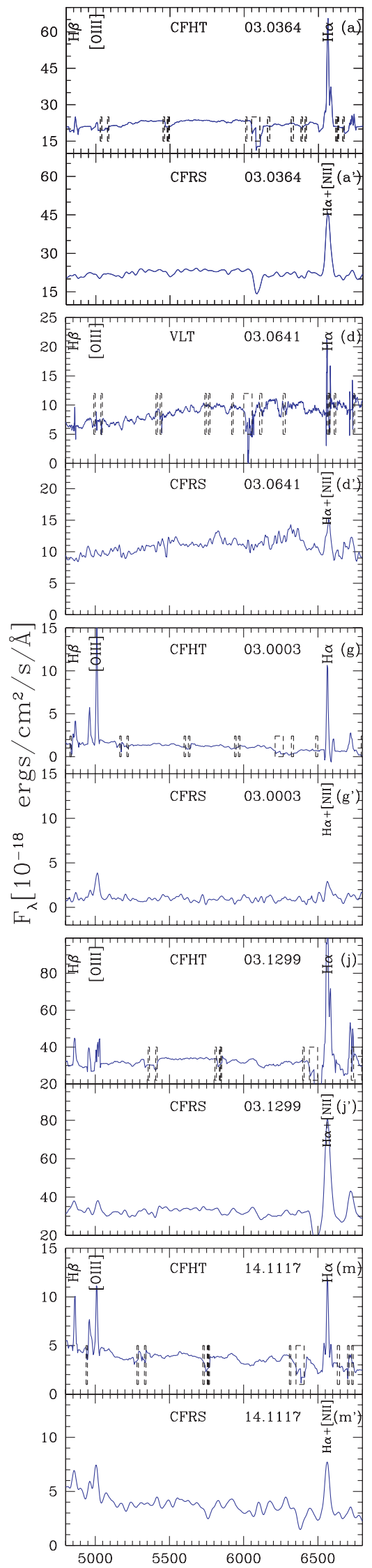
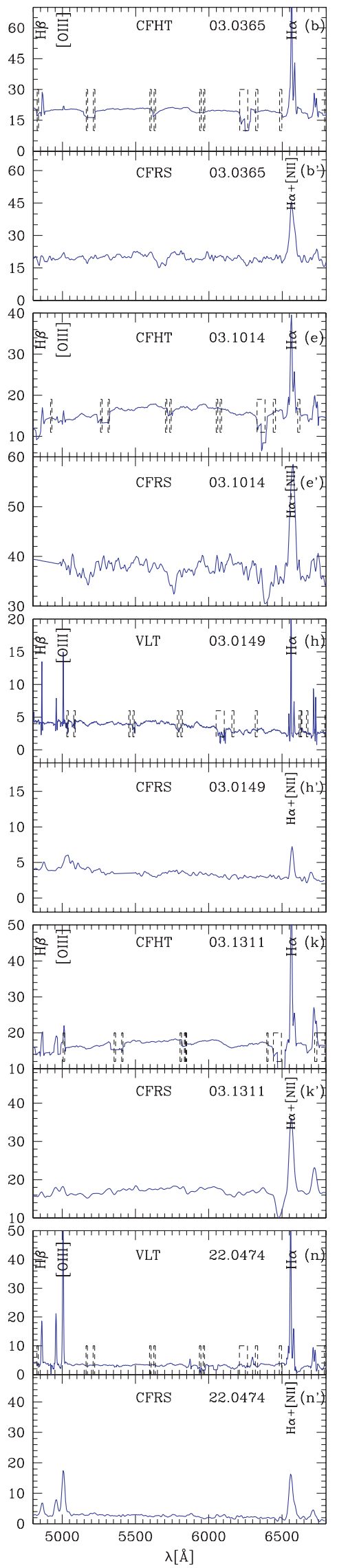
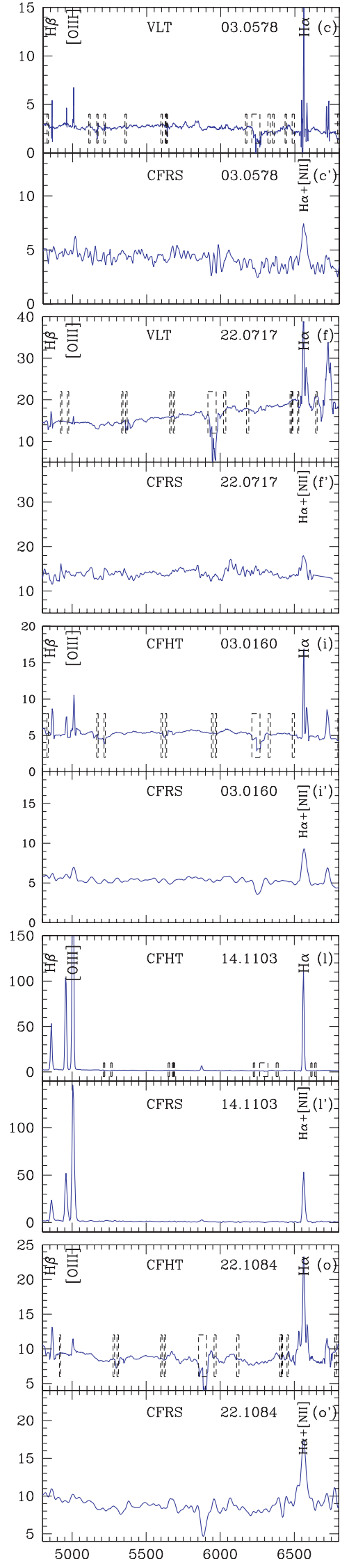

Fig. 2. a)-o) Rest-frame spectra of 15 sample galaxies from the moderate-resolution observations; $\mathbf{a}^{\prime}$ )-o' $\mathbf{o}^{\prime}$ ) the corresponding low-resolution CFRS spectra of the galaxies. a)-f) and $\left.\mathbf{a}^{\prime}\right)-\mathbf{f}^{\prime}$ ) are the spectra of the six "CFRS H $\alpha$-single" galaxies, others are the spectra of the nine "CFRS normal emission line" galaxies. The spectrum of another "CFRS H $\alpha$-single" galaxy, CFRS03.0711, will be given in Fig. 3. 
Table 2. Measured emission fluxes $\left(F_{\lambda}\right)$ of the sample galaxies in units of $10^{-17} \mathrm{ergs} \mathrm{cm}{ }^{-2} \mathrm{~s}^{-1}$, "9997" means the line is blended with strong sky line, "9998" means there are no the corresponding lines detected around the line positions, and "9999" means the lines are shifted outside the observed wavelength ranges. "Ap" is the aperture correction factor between the CFRS and the present moderate-resolution observations due to the different slit widths.

\begin{tabular}{|c|c|c|c|c|c|c|c|c|c|c|c|}
\hline CFRS & $\mathrm{H} \beta 4861$ & [O III] 4958 & [O III]5007 & $\mathrm{H} \alpha 6563$ & [N II]6548 & [N II]6583 & [S II]6716 & [S II]6731 & {$[\mathrm{S} \mathrm{II}]_{1+2}$} & [O II] 3727 & $A p$ \\
\hline 03.0364 & $7.18 \pm 0.57$ & $0.81 \pm 0.05$ & $2.44 \pm 0.14$ & $62.30 \pm 0.62$ & $6.89 \pm 0.41$ & $22.3 \pm 1.11$ & - & - & $10.90 \pm 1.50$ & 9999 & 1.0 \\
\hline 03.0365 & $13.49 \pm 0.54$ & $<0.54$ & $<1.61$ & $88.75 \pm 8.34$ & $14.25 \pm 1.43$ & $33.14 \pm 2.45$ & $12.55 \pm 1.04$ & $9.32 \pm 0.77$ & $21.87 \pm 1.81$ & $14.0 \pm 14.0$ & 1.0 \\
\hline 03.0578 & $1.23 \pm 0.20$ & $0.61 \pm 0.09$ & $1.77 \pm 0.26$ & $8.76 \pm 0.43$ & $1.06 \pm 0.34$ & $1.80 \pm 0.66$ & $1.80 \pm 0.31$ & $2.07 \pm 0.29$ & $3.87 \pm 0.60$ & $5.3 \pm 1.0$ & 1.8 \\
\hline 03.0641 & $2.21 \pm 0.24$ & $0.31 \pm 0.06$ & $0.92 \pm 0.17$ & $10.25 \pm 0.92$ & $1.18 \pm 0.14$ & $3.53 \pm 0.43$ & $<1.38$ & $<1.65$ & $<3.03$ & $3.0 \pm 1.0$ & 1.3 \\
\hline 03.0711 & $4.49 \pm 0.18$ & $0.65 \pm 0.07$ & $1.95 \pm 0.20$ & $13.58 \pm 0.58$ & $1.04 \pm 0.12$ & $5.78 \pm 0.68$ & $3.56 \pm 0.37$ & $1.96 \pm 0.37$ & $5.52 \pm 0.74$ & $2.5 \pm 2.0$ & 1.0 \\
\hline 03.1014 & $5.90 \pm 0.71$ & 9998 & $1.32 \pm 0.26$ & $43.28 \pm 4.51$ & $4.24 \pm 0.85$ & $13.00 \pm 1.71$ & $9.26 \pm 0.99$ & $5.12 \pm 2.78$ & $14.38 \pm 3.77$ & $16.0 \pm 7.0$ & 2.2 \\
\hline 22.0717 & $3.54 \pm 0.44$ & 9998 & $<0.75$ & $29.71 \pm 2.67$ & $3.67 \pm 0.40$ & $14.07 \pm 1.55$ & - & - & 9997 & $1.3 \pm 1.3$ & 1.0 \\
\hline 03.0003 & $3.97 \pm 0.80$ & $5.41 \pm 0.54$ & $16.16 \pm 0.86$ & $13.89 \pm 0.62$ & - & $<3.06$ & $2.80 \pm 0.20$ & $1.95 \pm 0.14$ & $4.75 \pm 0.50$ & $2.1 \pm 2.1$ & 0.6 \\
\hline 03.0149 & $4.76 \pm 0.28$ & $1.87 \pm 0.07$ & $5.43 \pm 0.22$ & $17.18 \pm 0.71$ & $1.35 \pm 0.17$ & $3.49 \pm 0.43$ & $3.95 \pm 0.72$ & $2.61 \pm 0.39$ & $6.56 \pm 1.11$ & $7.1 \pm 7.1$ & 1.0 \\
\hline 03.0160 & $4.25 \pm 0.51$ & $1.57 \pm 0.157$ & $4.72 \pm 0.47$ & $12.40 \pm 0.37$ & - & $6.17 \pm 0.66$ & - & - & $7.04 \pm 0.68$ & 9999 & 1.0 \\
\hline 03.1299 & $16.4 \pm 1.48$ & $5.06 \pm 0.46$ & $15.2 \pm 1.37$ & $144.0 \pm 4.32$ & $13.2 \pm 1.32$ & $39.6 \pm 3.96$ & - & - & $40.0 \pm 4.80$ & 9999 & 1.0 \\
\hline 03.1311 & $6.97 \pm 0.49$ & $2.53 \pm 0.20$ & $7.59 \pm 0.61$ & $58.4 \pm 2.34$ & $4.40 \pm 0.18$ & $13.4 \pm 0.54$ & $16.5 \pm 1.65$ & $10.5 \pm 1.05$ & $27.0 \pm 2.7$ & 9999 & 1.0 \\
\hline 14.1103 & $60.56 \pm 0.61$ & $130.9 \pm 0.52$ & $392.7 \pm 1.57$ & $133.9 \pm 0.54$ & 9998 & 9998 & - & - & 9998 & $<3.45$ & 1.0 \\
\hline 14.1117 & $5.54 \pm 0.44$ & $3.34 \pm 0.20$ & $10.03 \pm 0.60$ & $15.9 \pm 0.48$ & - & $2.70 \pm 0.54$ & - & - & $4.08 \pm 0.28$ & 9999 & 1.0 \\
\hline 22.0474 & $13.04 \pm 0.46$ & $14.05 \pm 0.30$ & $43.70 \pm 1.20$ & $51.97 \pm 2.49$ & $4.20 \pm 0.40$ & $11.97 \pm 2.0$ & $6.90 \pm 0.68$ & $5.80 \pm 0.58$ & $12.70 \pm 1.26$ & $17.0 \pm 2.3$ & 1.0 \\
\hline 22.1084 & $5.23 \pm 0.52$ & $0.94 \pm 0.12$ & $2.82 \pm 0.37$ & $26.9 \pm 2.15$ & $1.70 \pm 0.23$ & $5.11 \pm 0.71$ & - & - & $5.82 \pm 0.64$ & 9999 & 1.0 \\
\hline
\end{tabular}

Notes: $A p=\left(\frac{\text { CFRS }}{\text { VLT }}\right)_{\text {Aper }}$. [S II $]_{1+2}=[$ S II $] 6716+[\mathrm{S} \mathrm{II}] 6731$.

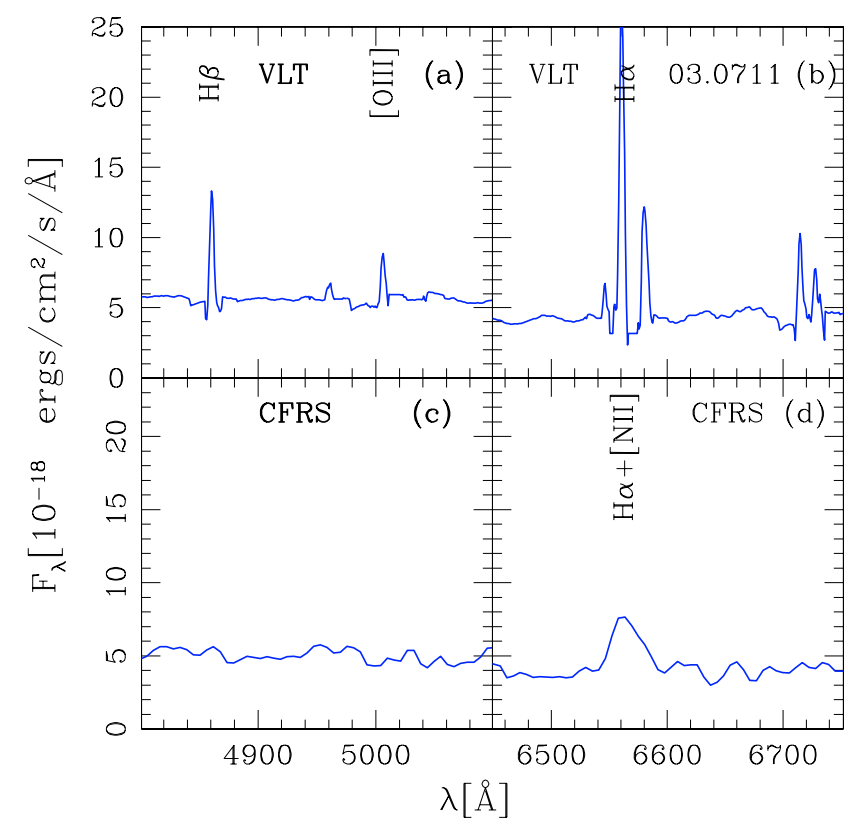

Fig. 3. a), b) Rest-frame spectra of CFRS03.0711 from the VLT observation around $\mathrm{H} \beta$ and $\mathrm{H} \alpha$ wavelength positions; c), d) the corresponding low-resolution CFRS spectra.
The extinction coefficient, $c$, can be derived using the Balmer lines $\mathrm{H} \alpha$ and $\mathrm{H} \beta$ :

$\left.\frac{I(\mathrm{H} \alpha)}{I(\mathrm{H} \beta)}=\frac{I_{0}(\mathrm{H} \alpha)}{I_{0}(\mathrm{H} \beta)} 10^{-c[f(\mathrm{H} \alpha)-f(\mathrm{H} \beta)}\right]$,

where $I(\mathrm{H} \alpha)$ and $I(\mathrm{H} \beta)$ are the measured integrated line fluxes, and $I_{0}(\mathrm{H} \alpha) / I_{0}(\mathrm{H} \beta)$ is the ratio of the fluxes as emitted in the interstellar dust. Assuming case B recombination, with a density of $100 \mathrm{~cm}^{-3}$ and a temperature of $10^{4} \mathrm{~K}$, the predicted ratio of $I_{0}(\mathrm{H} \alpha)$ to $I_{0}(\mathrm{H} \beta)$ is 2.86 (Osterbrock 1989).

Using the average interstellar extinction law given by Osterbrock (1989), $f(\mathrm{H} \alpha)-f(\mathrm{H} \beta)=-0.37, c$ can be readily determined from Eq. (1). Any corrected emission-line flux, $I_{0}(\lambda)$, can then be estimated by correcting the extinction obtained from Eq. (1) and the following average extinction law taken from Osterbrock (1989). The extinction parameter $A_{\mathrm{V}}$ (V for visual) has been calculated following the suggestion of Seaton (1979): $A_{\mathrm{V}}=E(B-V) R=\frac{c R}{1.47}$ (mag). $R$ which is 3.2, is the ratio of total to selective extinction at $\mathrm{V}$. The derived extinction values of the sample galaxies, $A_{\mathrm{V}}$, are given in Col. 2 in Table 3. Figure 4 shows the distributions of galaxy numbers vs. extinction $\left(A_{\mathrm{V}}\right)$ for the "CFRS $\mathrm{H} \alpha$-single", "CFRS normal emission line" galaxies and the combination of these two 
Table 3. The photometric properties of the sample galaxies.

\begin{tabular}{cccc}
\hline \hline CFRS & $A_{\mathrm{V}}$ & $\mathrm{B} / \mathrm{T}$ & $\begin{array}{c}\text { inclination } i \\
\text { (degree) }\end{array}$ \\
\hline 03.0364 & $2.84 \pm 0.21$ & $0.025_{-0.002}^{+0.002}$ & $11_{-1}^{+8}$ \\
03.0365 & $2.13 \pm 0.26$ & $0.010_{-0.010}^{+0.024}$ & $59_{-1}^{+1}$ \\
03.0578 & $2.33 \pm 0.44$ & $0.214_{-0.054}^{+0.047}$ & $55_{-3}^{+4}$ \\
03.0641 & $1.22 \pm 0.36$ & $0.007_{-0.007}^{+0.037}$ & $84_{-1}^{+1}$ \\
03.0711 & $0.14 \pm 0.14$ & $0.003_{-0.003}^{+0.013}$ & $32_{-3}^{+4}$ \\
03.1014 & $2.41 \pm 0.41$ & $0.021_{-0.003}^{+0.157}$ & $53_{-1}^{+4}$ \\
22.0717 & $2.75 \pm 0.39$ & $0.095_{-0.095}^{+0.089}$ & $72_{-2}^{+2}$ \\
\hline 03.0003 & $0.52 \pm 0.27$ & $0.868_{-0.434}^{+0.132}$ & $17_{-17}^{+33}$ \\
03.0149 & $0.59 \pm 0.18$ & $0.333_{-0.074}^{+0.150}$ & $51_{-3}^{+4}$ \\
03.0160 & $0.05_{-0.05}^{+0.30}$ & $0.036_{-0.036}^{+0.224}$ & $23_{-23}^{+54}$ \\
03.1299 & $2.87 \pm 0.24$ & $0.097_{-0.007}^{+0.071}$ & $59_{-1}^{+2}$ \\
03.1311 & $2.75 \pm 0.21$ & $0.038_{-0.013}^{+0.015}$ & $75_{-1}^{+1}$ \\
14.1103 & $0.00_{-0.00}^{+0.02}$ & $0.817_{-0.817}^{+0.183}$ & $39_{-13}^{+19}$ \\
14.1117 & $0.01_{-0.01}^{+0.22}$ & $0.147_{-0.147}^{+0.164}$ & $47_{-13}^{+7}$ \\
22.0474 & $0.85 \pm 0.13$ & $0.898_{-0.030}^{+0.020}$ & $27_{-3}^{+4}$ \\
22.1084 & $1.49 \pm 0.32$ & $0.000_{-0.000}^{+0.054}$ & $2_{-2}^{+8}$ \\
\hline
\end{tabular}

with a 0.5 bin in $A_{\mathrm{V}}$. "CFRS $\mathrm{H} \alpha$-single" galaxies mostly display high extinction coefficients (median $A_{\mathrm{V}}=2.2$ ), conversely to "CFRS normal emission line" galaxies (median $A_{\mathrm{V}}=0.6$ ). However, taking all the 16 galaxies together leads to an average extinction coefficient of about $A_{\mathrm{V}}=1.25$, which is equivalent to the assumption of Fujita et al. (2003, $\left.A_{\mathrm{H} \alpha}=1.0\right)$. No very high extinctions $\left(A_{\mathrm{V}}>3.3\right)$ are found in the sample galaxies.

\subsection{Photometry and dust correction}

Using the HST and the CFHT images of the sample galaxies, we used the GIM2D ${ }^{2}$ software package to calculate the inclination $i$ (the disk axis to the line of sight) and the luminosities ratio of the bulge over the total $(\mathrm{B} / \mathrm{T})$ of these galaxies. Table 3 gives the corresponding values. All "CFRS H $\alpha$-single" galaxies show disk properties with $\mathrm{B} / \mathrm{T}$ ratios lower than 0.5 . The $\mathrm{B} / \mathrm{T}$ values are consistent with the study of Kent (1985), which showed that the $\mathrm{B} / \mathrm{T}$ ratio is mainly between 0.4 to 0.0 for

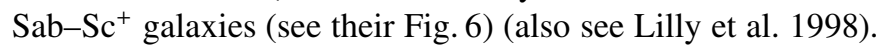
Three "CFRS normal emission line" galaxies (CFRS03.0003, 14.1103 and 22.0474) are very compact and the analysis of their CFHT images hardly recover their morphological parameters (which results in large error bars).

\footnotetext{
${ }^{2}$ GIM2D, Galaxy Image 2D, is an IRAF/SPP package written to perform detailed bulge+disk surface brightness profile decompositions of low signal-to-noise images of distant galaxies in a fully automated way (Simard et al. 2002).
}

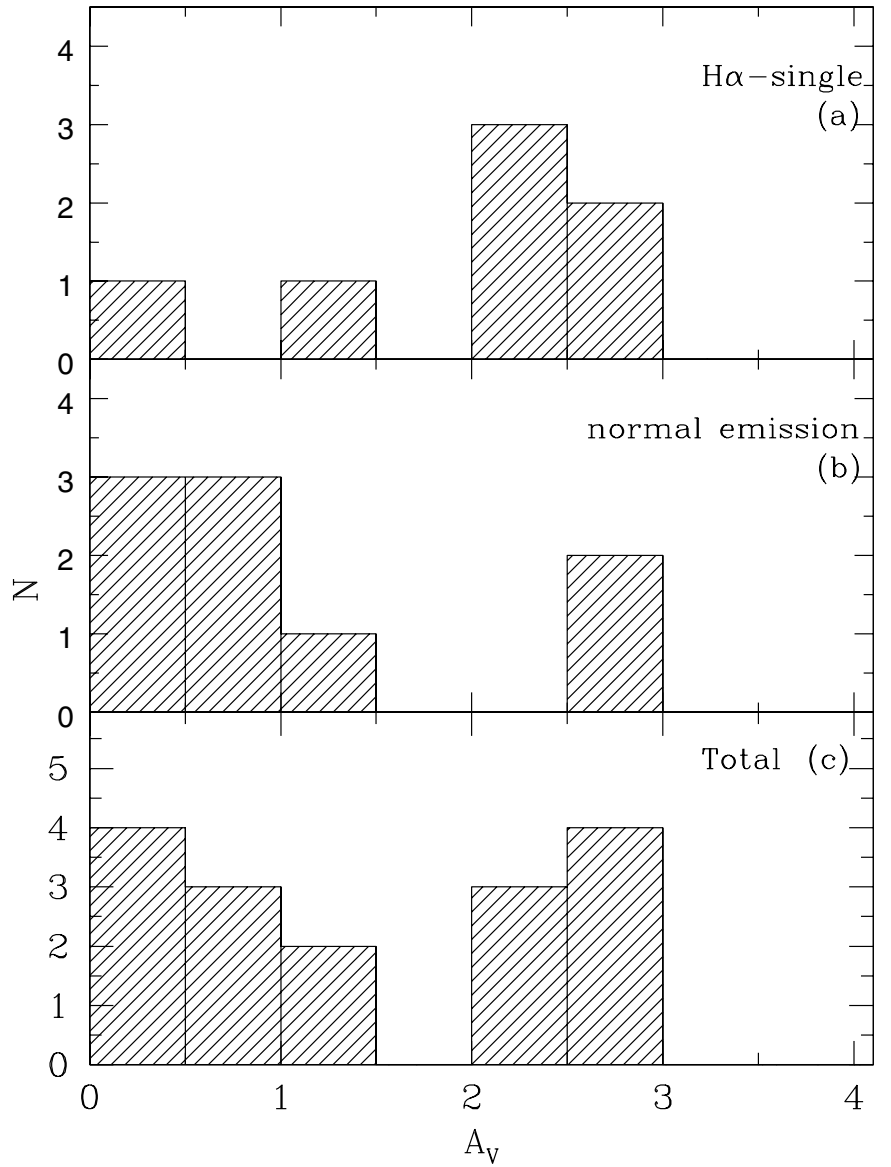

Fig. 4. Distribution of the extinction $A_{\mathrm{V}}$ in sample galaxies with a binning interval of 0.5 : a) the seven "CFRS $\mathrm{H} \alpha$-single" galaxies; b) the nine "CFRS normal emission line" galaxies; c) the total 16 sample galaxies.

Generally, the internal extinction of galaxies increases with the inclination of the disk because the path length through the disk increases roughly as $1 / \cos i$ (Giovanelli et al. 1995). Figure 6 provides a good illustration of this effect, since for galaxies with inclination lower than $45^{\circ}$, the median $A_{\mathrm{V}}$ is 0.6 which could be compared to 2.2 in the edge-on galaxies.

\section{Diagnostic diagrams and gas abundances}

\subsection{Diagnostic diagrams}

Several emission line ratios have been used for a proper diagnostic for the galaxies. Figures $7 \mathrm{a}$ and $7 \mathrm{~b}$ give the diagnostic diagrams of $\log ([\mathrm{O}$ III $] \lambda 5007 / \mathrm{H} \alpha)$ vs. $\log ([\mathrm{N} \mathrm{III}] \lambda 6583 / \mathrm{H} \alpha)$ and $\log ([\mathrm{O} \mathrm{III}] \lambda 5007 / \mathrm{H} \alpha)$ vs. $\log ([\mathrm{S} \mathrm{II}] \lambda 6716+\lambda 6731 / \mathrm{H} \alpha)$, respectively.

The $[\mathrm{O} \mathrm{III}] / \mathrm{H} \beta$ ratio is mainly an indicator of the mean level of ionization and temperature, while the $[\mathrm{S}$ II $] / \mathrm{H} \alpha$ ratio is an indicator of the relative importance of a large partially ionized zone produced by high-energy photoionization. The $[\mathrm{N} \mathrm{II}] / \mathrm{H} \alpha$ ratio also gives a good separation between $\mathrm{HII}$ region nuclei and Active Galactic Nuclei (AGN) though its significance is not so immediately obvious. The ratios have been chosen to minimize the effects of dust extinction (Veilleux \& Osterbrock 1987; Osterbrock 1989). 


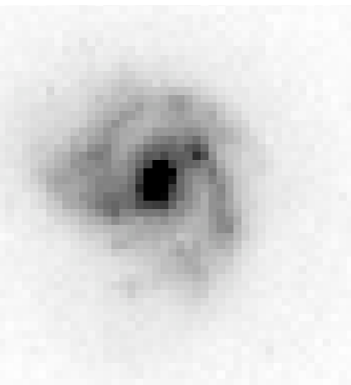

(a) 03.0364, HST;

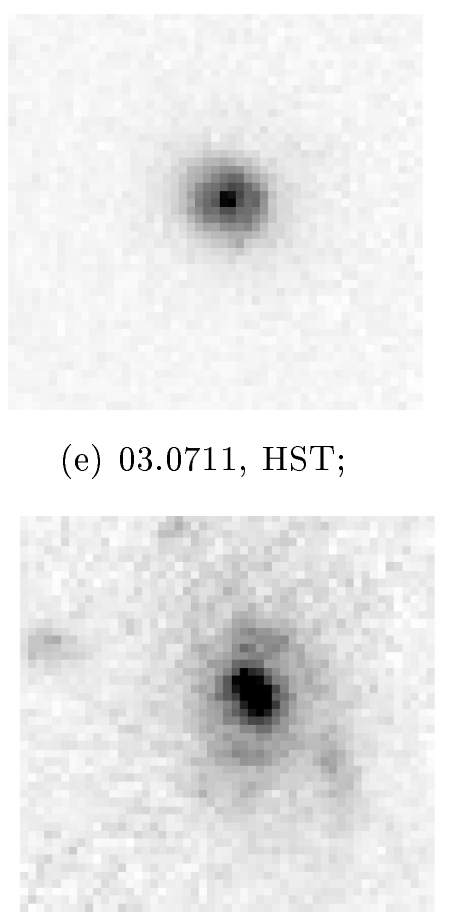

(i) 03.0149 , HST;

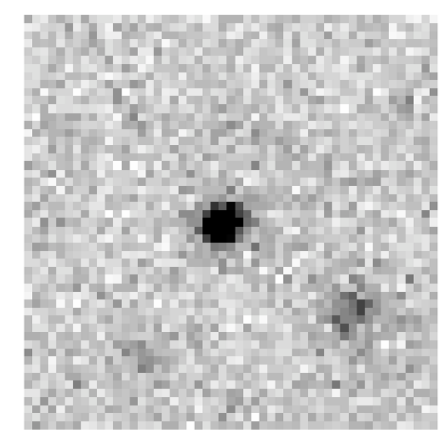

(m) 14.1103, CFHT;
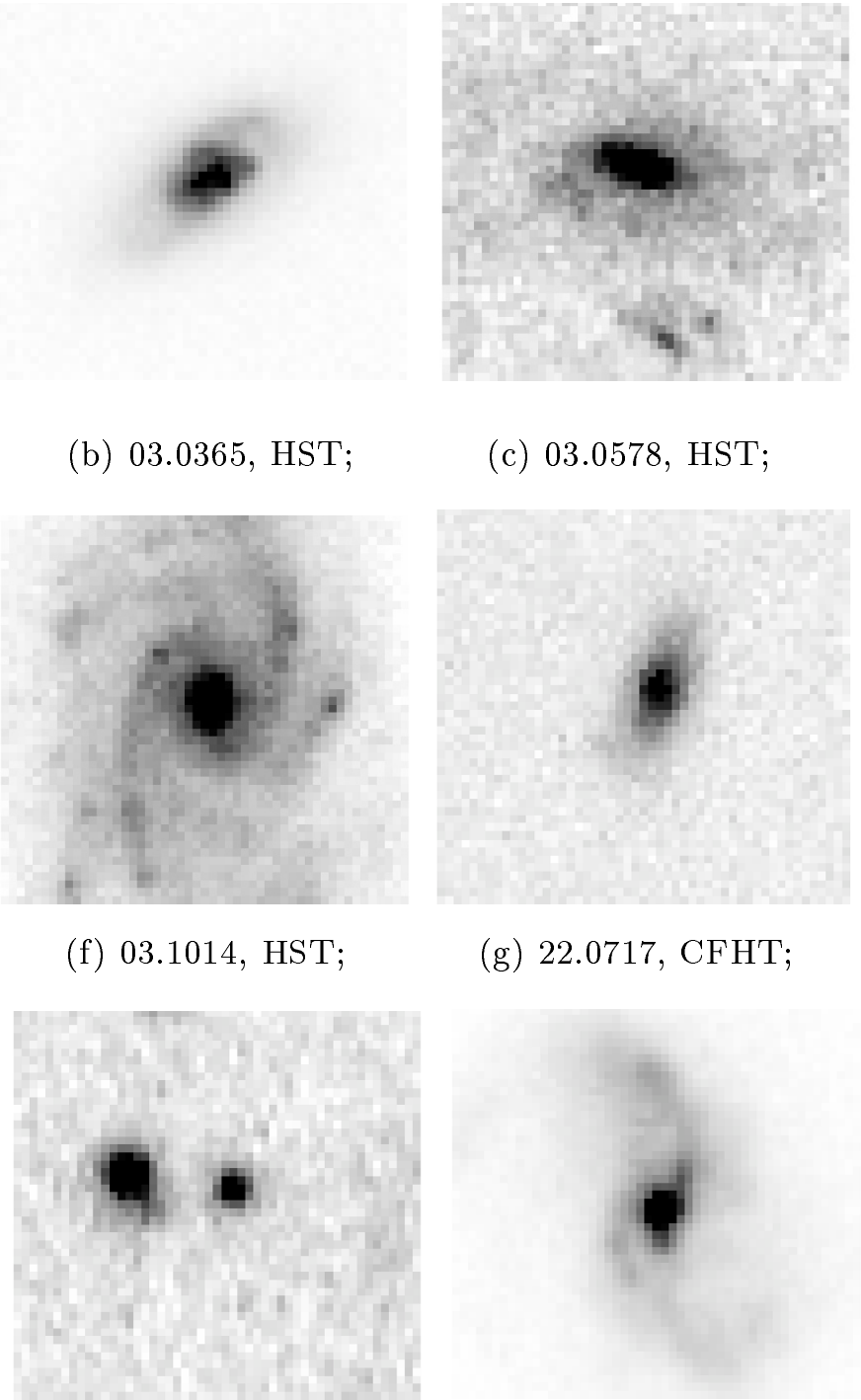

(j) 03.0160, CFHT;

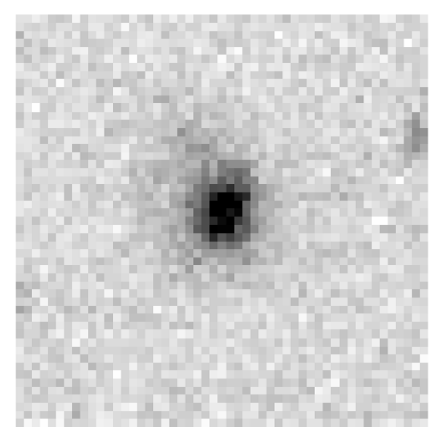

(n) 14.1117, CFHT; (c) 03.0578, HST;

(g) 22.0717, CFHT;

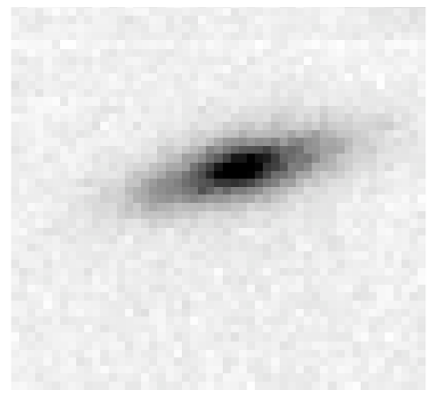

(d) 03.0641, CFHT;

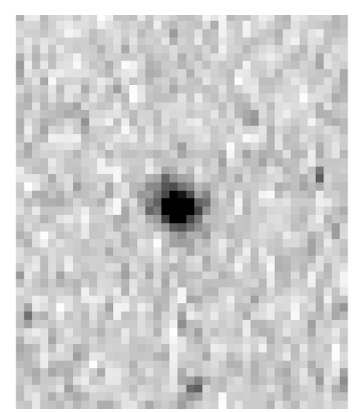

(h) 03.0003, CFHT;

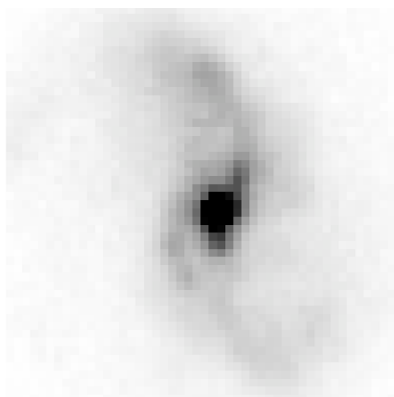

(k) 03.1299, HST;

(1) 03.1311, HST;

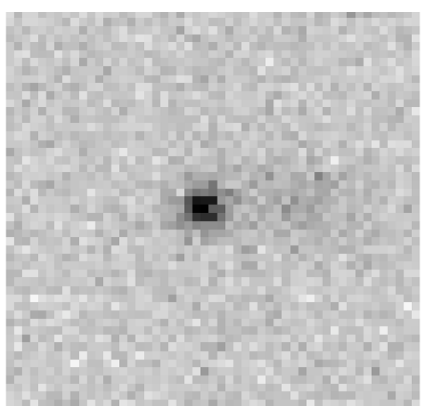

(o) 22.0474, CFHT;

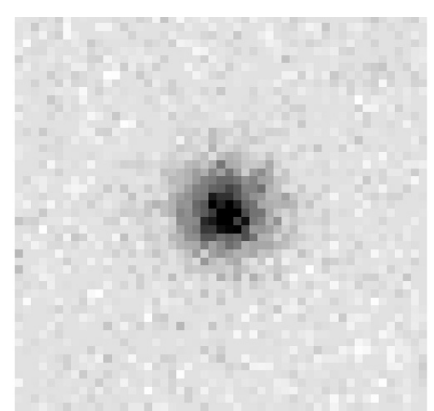

(p) 22.1084, CFHT.

Fig. 5. HST/F814W or CFHT images of the sample galaxies. a)-g) are the images of the seven "CFRS H $\alpha$-single" galaxies; the rest nine are "CFRS normal emission line" galaxies. Images are 5 " $\times 5$ " size, only 03.0003 is 4 " in the horizontal size since it is near to the edge of the image of the field.

The dashed and dotted lines in the two diagrams are the separating lines between $\mathrm{HII}$ region nuclei and AGNs taken from Osterbrock (1989, their Figs. 12.1 and 12.3). The HII regionlike objects can be H II regions in external galaxies, starbursts, or H II region galaxies, objects known to be photoionized by OB stars. Seyfert 2 galaxies have relatively high ionization with [O III] $\lambda 5007 / \mathrm{H} \beta \geq 3$. Most starburst and H II region galaxies have lower ionization. Many low-ionization galaxies have 


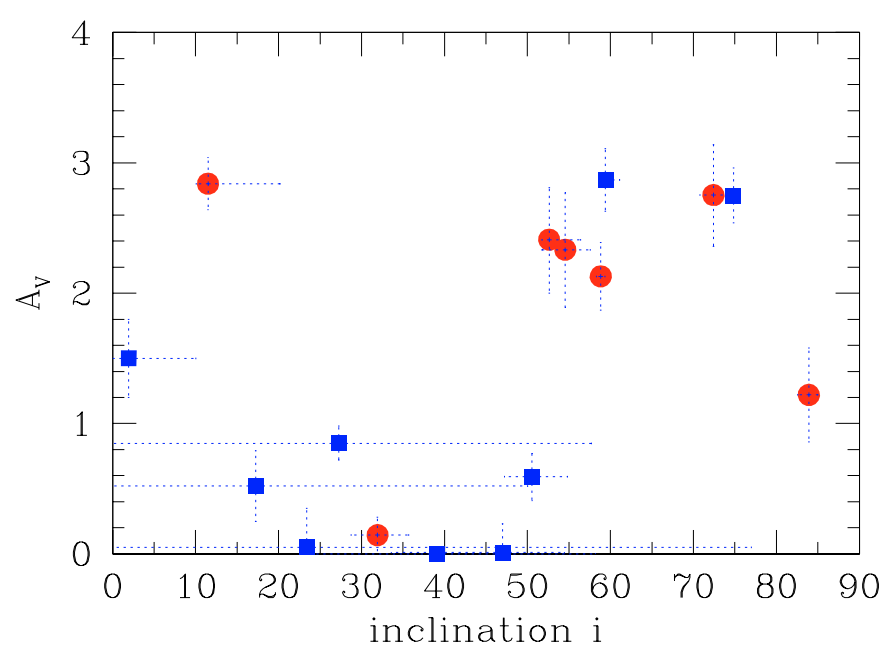

Fig. 6. The relations between extinction $A_{\mathrm{V}}$ and inclination $i$ in the sample galaxies. Symbols as in Fig. 1.

stronger [S II] $\lambda \lambda 6716,6731$ and [N II] $\lambda 6583$ than H II regions or starburst galaxies. These objects have been named "LowIonization Nuclear Emission-line Regions" (LINERs).

From Fig. $7 \mathrm{a}$, it seems that all of the sample galaxies are H II-region galaxies except for the possible "LINER" property of CFRS03.0160 due to the strong [N II] emission. Figure 7b confirms again that most of the studied galaxies lie in the H II-region locus though few of them are in the active region locus (LINER or Seyfert), including CFRS03.0160. Shock-wave ionization may produce stronger [S II] $\lambda \lambda 6716,6731$ emissions relative to $\mathrm{H} \alpha$ than in typical $\mathrm{H}$ II regions. For some of the best quality spectra, we have been able to estimate the intensity ratio of the two [S II] emission line, ([S II] $]$ 6716)/([S II $] \lambda 6731)$ which can be used to estimate the electron density, $N_{\mathrm{e}}$ (Osterbrock 1989, p. 134, their Fig. 5.3). It results the values which are generally close to what is expected from H II region galaxies (may also see van Zee et al. 1998).

From the combination of Figs. 7a and 7b, we find that it seems that only one object (CFRS03.0160) out of 16 could be a LINER. Moderate resolution spectroscopy is required to establish these two diagnostic diagrams, and is unique for solid estimates of the nature of emission line objects. As also noticed by Tresse et al. (1996), a large fraction of their assumed Seyfert 2 galaxies would be better classified as LINERs if the underlying absorption under the $\mathrm{H} \beta$ line was properly accounted for.

In both Figs. 7a and 7b, the "CFRS $\mathrm{H} \alpha$-single" galaxies and the "CFRS normal emission line" galaxies lie in well distinct areas, with the noticeable exception of CFRS03.0578. We believe this is related to different gas metal abundance histories in these two classes of galaxies as it is studied in the following sections.

\subsection{Metallicities from comparison with local $H I I$ regions and $H I I$ galaxies}

Gas abundance is a key factor to understand the star formation history and stellar population components of galaxies. Metallicities of galaxies can be roughly estimated from the
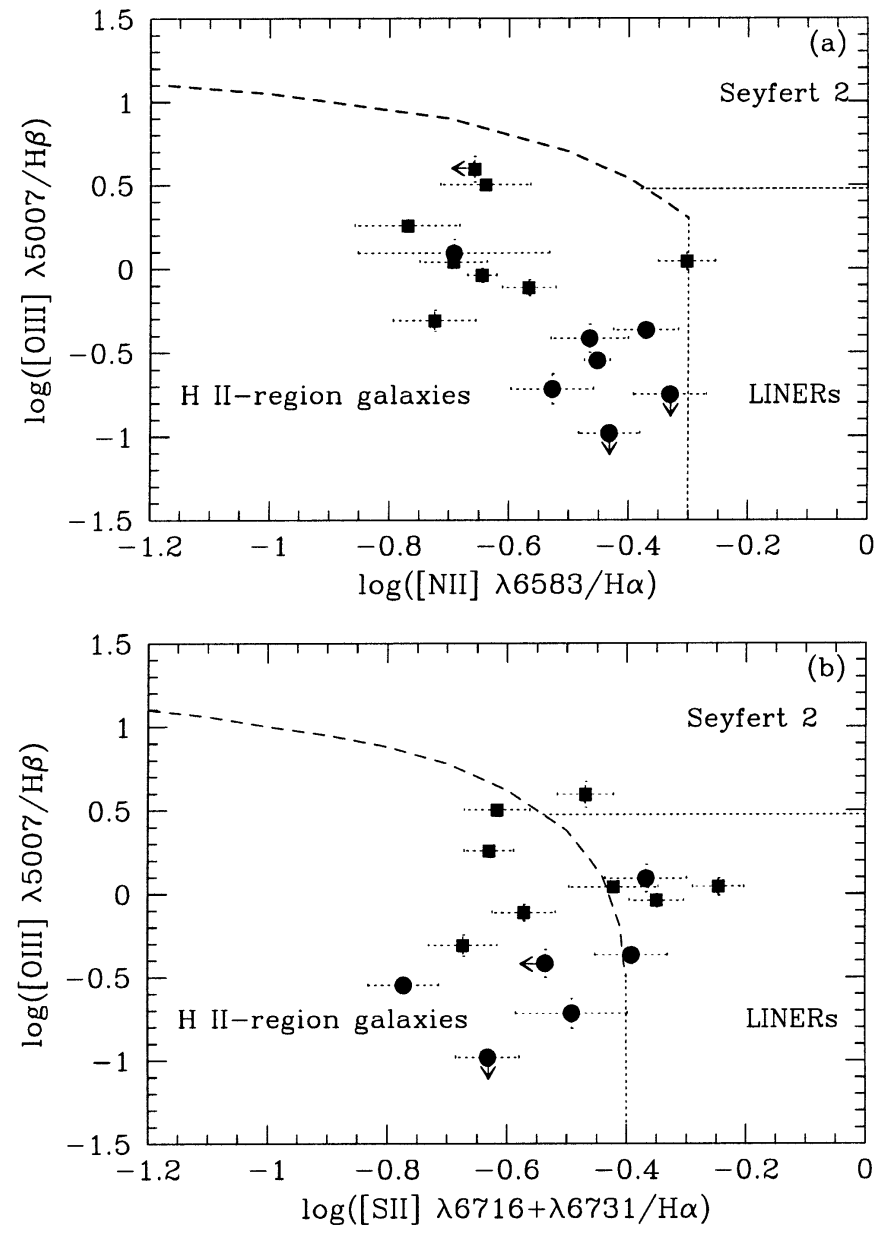

Fig. 7. a) $[\mathrm{OIII}] / \mathrm{H} \beta$ vs. $[\mathrm{N} \mathrm{II}] / \mathrm{H} \alpha$ diagnostic diagram for the sample galaxies; b) $[\mathrm{O} \mathrm{III}] / \mathrm{H} \beta$ vs. $[\mathrm{S} \mathrm{II}] / \mathrm{H} \alpha$ diagnostic diagram for the sample galaxies. Symbols as in Fig. 1.

diagnostic diagram of $[\mathrm{O} \mathrm{II}] / \mathrm{H} \beta$ vs. $[\mathrm{O} \mathrm{III}] / \mathrm{H} \beta$ by comparing with the known metallicities of the local HII regions and H II galaxies (Hammer et al. 1997).

Figure 8 gives the $\log ([\mathrm{O}$ II $] \quad \lambda 3727 / \mathrm{H} \beta) \quad$ vs. $\log ([\mathrm{O}$ III $] \lambda 4958+\lambda 5007 / \mathrm{H} \beta)$ relations in "CFRS $\mathrm{H} \alpha$-single" galaxies (the filled circles) and "CFRS normal emission line" galaxies (the filled squares), associated with a sample of local H II regions and HII galaxies with known metallicities. The data points of other galaxies are taken from the literature (see Hammer et al. 1997 for references). It shows that the "CFRS $\mathrm{H} \alpha$-single" galaxies are more metal-rich than the "normal emission line" galaxies except CFRS03.0578, which results in weaker [O II] and [O III] emissions.

Since [O II] emission lines are outside the rest-frame wavelength ranges in six galaxies ("9999" were marked for their fluxes of [O II] emission in Table 2), we use the theoretical $[\mathrm{O}$ II $] / \mathrm{H} \beta$ values of the local $\mathrm{H} \mathrm{II}$ regions and $\mathrm{H} \mathrm{II} \mathrm{galaxies} \mathrm{(the}$ solid line in the figure) to be their $[\mathrm{O} \mathrm{II}] / \mathrm{H} \beta$ values to estimate the metallicities, which is reliable by virtue of their H II-region galaxy properties given in Fig. 7 and the reliable $[\mathrm{O}$ III] $] / \mathrm{H} \beta$ ratios. Actually, the $[\mathrm{O} \mathrm{III}] / \mathrm{H} \beta$ ratio values give the metallicities of these galaxies by comparing with the corresponding ratios of the local HII regions and HII galaxies with different 


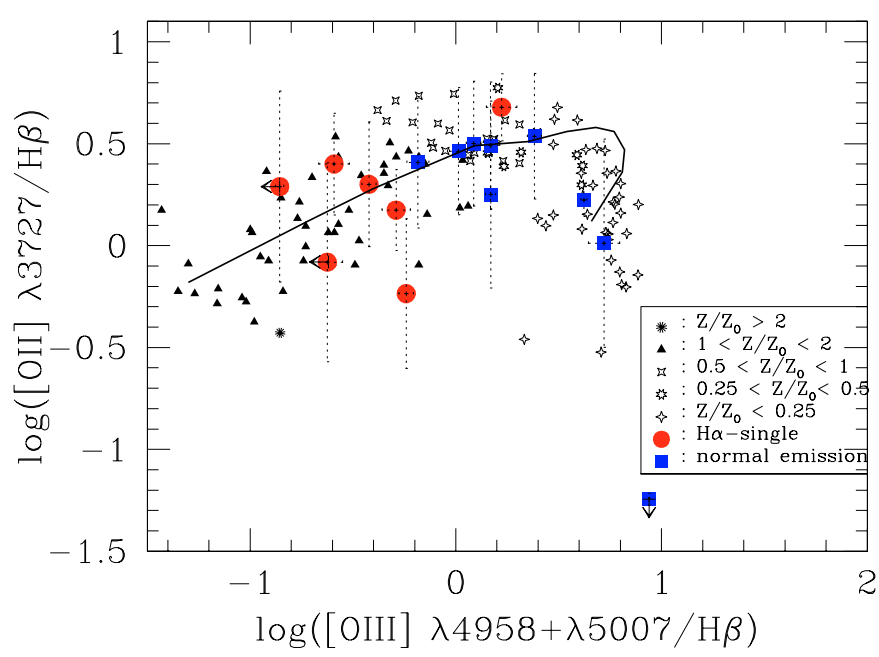

Fig. 8. The relations between $[\mathrm{O} \mathrm{II}] / \mathrm{H} \beta$ and $[\mathrm{O} \mathrm{III}] / \mathrm{H} \beta$ in the "CFRS $\mathrm{H} \alpha$-single" galaxies (filled circles) and the "CFRS normal emission line" galaxies (filled squares), together with a sample of the local $\mathrm{HII}$ regions and HII galaxies with different metallicities. The solid line shows the theoretical sequence from McCall et al. (1985), which fits the local H II galaxies well, with metallicity decreasing from the left to the right (also see Hammer et al. 1997).

metallicities in Fig. 8. Their error bars of [O II] fluxes are estimated by using the average error of other sample galaxies.

\subsection{Oxygen and nitrogen abundances}

Oxygen is one of the main coolants in nebulae, appearing either via fine-structure lines in the far-infrared (52 and $88 \mu \mathrm{m}$ ) when the electron temperature is low or via forbidden lines in the

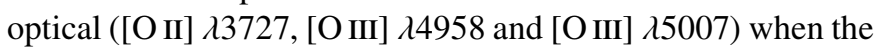
electron temperature is high.

Given the absence of reliable [O III] $\lambda 4363$ detection, which is too weak to be measured except in extreme metal-poor galaxies, to derive the electron temperature of the ionized medium by comparing with [O III] $\lambda \lambda 4958,5007$ lines (Osterbrock 1989), oxygen abundances may also be determined from the ratio of $[\mathrm{O} \mathrm{II}]+[\mathrm{O} \mathrm{III}]$ to $\mathrm{H} \beta$ lines ("strong line" method). The general parameter is $R_{23}: R_{23}=([\mathrm{O}$ II $] \lambda 3727+[\mathrm{O}$ III] $\lambda 4958+$ [O III] $\lambda 5007) / \mathrm{H} \beta$. To convert $R_{23}$ into $12+\log (\mathrm{O} / \mathrm{H})$, we adopt the analytical approximation given by Zaritsky et al. (1994, hereafter $\mathrm{ZKH}$ ), which is consistent with other calibration relations (see Kobulnicky \& Zaritsky 1999, KZ99), and that is itself a polynomial fit to the average of three earlier calibrations for metal rich HII regions. This relationship has been used for all the galaxies except for CFRS14.1103 which presents a very small $[\mathrm{O}$ II $] / \mathrm{H} \beta$ ratio and no $[\mathrm{N}$ II] $\lambda \lambda 6548,6583$ and [S II] $\lambda \lambda 6716,6731$ emission lines are detected that characterize a low oxygen abundance medium, and for which we adopt the relation from Kobulnicky et al. (1999) for metal-poor branch galaxies. Its derived abundance by us is very similar to that of Tresse et al. (1993).

The derived oxygen abundances of the sample galaxies are given in Table 4 as $12+\log (\mathrm{O} / \mathrm{H})$, which are consistent with the results of Fig. 8. The "CFRS $\mathrm{H} \alpha$-single" galaxies have larger abundance values than those of "CFRS normal emission line" galaxies, and lie in a region occupied by over-solar abundance objects, except for CFRS03.0578.

Figure 9 shows the oxygen abundance vs. absolute blue magnitude $M_{B}$ relations for the sample galaxies. It shows that the "CFRS H $\alpha$-single" galaxies are lying in the top metallicity area of local spiral galaxies and Emission Line Galaxies (ELGs) at $z=0.1 \sim 0.5$ studied by KZ99, and the "CFRS normal emission line" galaxies are very similar to the local spiral galaxies except CFRS14.1103. The general trend of the sample galaxies is that the brighter galaxies are more metal-rich. The Solar oxygen abundance $\left(12+\log (\mathrm{O} / \mathrm{H})_{\odot}=8.83\right)$ was taken from Grevesse \& Sauval (1998).

[N II] $\lambda 6583$ can be used in conjunction with [O II] $\lambda 3727$ and the temperature in the $[\mathrm{N} \mathrm{II}]$ emission regions $\left.\left(t_{[\mathrm{N} \text { II }}\right]\right)$ to estimate the $\mathrm{N} / \mathrm{O}$ ratio in the sample galaxies assuming $\frac{\mathrm{N}}{\mathrm{O}}=\frac{\mathrm{N}^{+}}{\mathrm{O}^{+}}$. Uncertainties due to emission line measurements, reddening and sky subtraction in the presence of strong night sky emission lines near [N II] dominate the error budget for N/O.

First, we use the formula given by Thurston et al. (1996) to estimate the temperature in the $\left[\mathrm{N}\right.$ II] emission region $\left.\left(t_{[\mathrm{N} \text { II }}\right]\right)$ by using $\log R_{23}$. Then, $\log (\mathrm{N} / \mathrm{O})$ is estimated from ([N II] $\lambda 6548+$ 16583)/([O II $]$ 13727) emission ratio and $\left.t_{[\mathrm{NII}}\right]$. The derived values are given in Table 4. Figure 10 gives the $\log (\mathrm{N} / \mathrm{O})$ vs. $12+\log (\mathrm{O} / \mathrm{H})$ relations for these sample galaxies. Most of them follow the secondary nitrogen production well (Vila-Costas \& Edmunds 1993).

\section{6. $\mathrm{H} \alpha$ luminosities and star formation rates}

\subsection{Ho luminosities and SFRs}

There is a unique advantage in using $\mathrm{H} \alpha$ to obtain the SFRs for low- $z$ galaxies. Among the Balmer lines, $\mathrm{H} \alpha$ is the most directly proportional to the ionizing UV flux, and the weaker Balmer lines are much more affected by stellar absorption and reddening. The SFRs of the "CFRS H $\alpha$-single" galaxies were estimated from the $\mathrm{H} \alpha$ luminosities.

In the following, we adopt a Salpeter initial mass function (IMF) with low and high mass cutoffs at 0.1 and $100 M_{\odot}$ (Salpeter 1955). The calibrations of Kennicutt et al. (1994) and Madau et al. (1998) yield:

$\operatorname{SFR}\left(M_{\odot} \mathrm{yr}^{-1}\right)=7.9 \times 10^{-42} L(\mathrm{H} \alpha)\left(\operatorname{ergs~s}^{-1}\right) \times$ Aper,

(Kennicutt 1998), with

$L(\mathrm{H} \alpha)=4 \pi\left(3.086 \times 10^{24} D_{\mathrm{L}}\right)^{2} f(\mathrm{H} \alpha)\left(\mathrm{ergs} \mathrm{s}^{-1}\right)$,

where $L(\mathrm{H} \alpha)$ is the $\mathrm{H} \alpha$ luminosity in ergs $\mathrm{s}^{-1}, f(\mathrm{H} \alpha)$ is the integrated flux in ergs s $\mathrm{cm}^{-1} \mathrm{~cm}^{-2}$ after correcting for the extinction, and $D_{\mathrm{L}}$ is the luminosity distance in Mpc. Aper is the aperture correction factor by comparing the photometric and spectral magnitudes in $I_{\mathrm{AB}}$ band due to the limited size of the slit. The related results of these parameters and the derived SFRs are given in the left part of Table 5 (Cols. (1)-(5)), in which $\operatorname{Flux}_{\mathrm{c}}(\mathrm{H} \alpha)$ is the $\mathrm{H} \alpha$ emission flux after correcting for the extinction. CFRS galaxies have SFRs ranging from Milky Way value to higher typical values of starburst galaxies. 
Table 4. The derived emission line ratios and oxygen, nitrogen abundances of the sample galaxies. The first seven are the "CFRS H $\alpha$-single" galaxies, and the rest nine are the "CFRS normal emission line" galaxies.

\begin{tabular}{|c|c|c|c|c|c|c|c|c|c|c|}
\hline CFRS & $\log \frac{\left[\mathrm{O}_{\mathrm{IIII}}\right]}{\mathrm{H} \beta}$ & $\log \frac{\left[\mathrm{NII}_{\mathrm{II}}\right.}{\mathrm{H} \alpha}$ & $\log \frac{\left[\mathrm{S}_{\mathrm{II}}\right]}{\mathrm{H} \alpha}$ & $\log \frac{\left[\mathrm{O}_{\mathrm{III}}\right]}{\mathrm{H} \beta}$ & $\frac{\left[\mathrm{SII}_{1}\right]_{1}}{[\mathrm{SII}]_{2}}$ & $\begin{array}{r}N_{\mathrm{e}} \\
\left(\mathrm{cm}^{-3}\right)\end{array}$ & $\log \left(R_{23}\right)$ & $12+\log (\mathrm{O} / \mathrm{H})$ & $\left.t_{\left[\mathrm{N}_{\mathrm{II}}\right]}\right](\mathrm{K})$ & $\log (\mathrm{N} / \mathrm{O})$ \\
\hline 03.0364 & $-0.55 \pm 0.04$ & $-0.45 \pm 0.02$ & $-0.77 \pm 0.06$ & $0.30 \pm 0.30$ & - & - & $0.38 \pm 0.26$ & $9.09 \pm 0.17$ & $7082 \pm 1095$ & $-0.90 \pm 0.01$ \\
\hline 03.0365 & $<-0.98$ & $-0.43 \pm 0.05$ & $-0.63 \pm 0.05$ & $0.29 \pm 0.47$ & $1.35 \pm 0.22$ & $\sim 10^{2}$ & $<0.32$ & $>9.13$ & $<6861$ & $\sim-0.86$ \\
\hline 03.0578 & $0.09 \pm 0.08$ & $-0.69 \pm 0.16$ & $-0.37 \pm 0.07$ & $0.68 \pm 0.22$ & $0.87 \pm 0.27$ & $\sim 9 \times 10^{2}$ & $0.81 \pm 0.13$ & $8.61 \pm 0.23$ & $10074 \pm 1343$ & $-1.12 \pm 0.18$ \\
\hline 03.0641 & $-0.42 \pm 0.08$ & $-0.47 \pm 0.04$ & $<-0.54$ & $0.17 \pm 0.24$ & $0.84 \pm 0.53$ & $\sim 10^{3}$ & $0.30 \pm 0.16$ & $9.13 \pm 0.09$ & $6798 \pm 571$ & $-0.82 \pm 0.19$ \\
\hline 03.0711 & $-0.37 \pm 0.04$ & $-0.37 \pm 0.05$ & $-0.39 \pm 0.06$ & $-0.24 \pm 0.37$ & $1.81 \pm 0.54$ & $<10^{1}$ & $0.06 \pm 0.20$ & $9.24 \pm 0.07$ & $6173 \pm 375$ & $-0.47 \pm 0.38$ \\
\hline 03.1014 & $-0.7 \pm 0.10$ & $-0.53 \pm 0.07$ & $-0.49 \pm 0.09$ & $0.40 \pm 0.48$ & $1.81 \pm 0.70$ & $<10^{1}$ & $0.44 \pm 0.23$ & $9.05 \pm 0.17$ & $7384 \pm 1135$ & $-1.02 \pm 0.58$ \\
\hline 22.0717 & $<-0.75$ & $-0.33 \pm 0.06$ & $0.07 \pm 0.05$ & $-0.08 \pm 0.49$ & - & - & $<0.03$ & $>9.25$ & $<6111$ & $\sim-0.57$ \\
\hline 03.0003 & $0.60 \pm 0.08$ & $<-0.66$ & $-0.47 \pm 0.05$ & $0.01 \pm 0.34$ & - & - & $0.80 \pm 0.12$ & $8.63 \pm 0.21$ & $<9965$ & $<-0.63$ \\
\hline 03.0149 & $0.04 \pm 0.03$ & $-0.69 \pm 0.06$ & $-0.42 \pm 0.07$ & $0.25 \pm 0.46$ & $1.51 \pm 0.50$ & $<10^{1}$ & $0.51 \pm 0.26$ & $8.99 \pm 0.22$ & $7756 \pm 1465$ & $-0.97 \pm 0.56$ \\
\hline 03.0160 & $0.04 \pm 0.05$ & $-0.30 \pm 0.05$ & $-0.25 \pm 0.04$ & $0.49 \pm 0.31$ & - & - & $0.66 \pm 0.22$ & $8.83 \pm 0.28$ & $8752 \pm 1720$ & $-0.86 \pm 0.01$ \\
\hline 03.1299 & $-0.11 \pm 0.05$ & $-0.57 \pm 0.04$ & $-0.57 \pm 0.05$ & $0.46 \pm 0.31$ & - & - & $0.60 \pm 0.24$ & $8.91 \pm 0.25$ & $8273 \pm 1621$ & $-1.02 \pm 0.01$ \\
\hline 03.1311 & $-0.04 \pm 0.04$ & $-0.64 \pm 0.02$ & $-0.35 \pm 0.05$ & $0.50 \pm 0.30$ & $1.58 \pm 0.20$ & $<10^{1}$ & $0.64 \pm 0.23$ & $8.86 \pm 0.27$ & $8612 \pm 1707$ & $-1.10 \pm 0.02$ \\
\hline 14.1103 & $0.81 \pm 0.00$ & 9998 & 9998 & $<-1.24$ & - & - & $0.94 \pm 0.00$ & $<7.63$ & $<11583$ & \\
\hline 14.1117 & $0.26 \pm 0.04$ & $-0.77 \pm 0.09$ & $-0.63 \pm 0.04$ & $0.54 \pm 0.31$ & - & - & $0.77 \pm 0.19$ & $8.68 \pm 0.30$ & $9664 \pm 1782$ & $-1.17 \pm 0.03$ \\
\hline 22.0474 & $0.50 \pm 0.02$ & $-0.61 \pm 0.07$ & $-0.62 \pm 0.05$ & $0.22 \pm 0.08$ & $1.19 \pm 0.23$ & $\sim 3 \times 10^{2}$ & $0.77 \pm 0.03$ & $8.68 \pm 0.05$ & $9679 \pm 285$ & $-0.72 \pm 0.06$ \\
\hline 22.1084 & $-0.31 \pm 0.06$ & $-0.72 \pm 0.07$ & $-0.67 \pm 0.06$ & $0.41 \pm 0.32$ & - & - & $0.51 \pm 0.26$ & $8.99 \pm 0.23$ & $7727 \pm 1507$ & $-1.18 \pm 0.01$ \\
\hline
\end{tabular}

Notes: $\log \frac{[\mathrm{OIII}]}{\mathrm{H} \beta}=\log \frac{[\mathrm{OIII}] 5007}{\mathrm{H} \beta}, \log \frac{[\mathrm{NII}]}{\mathrm{H} \alpha}=\log \frac{[\mathrm{NII}]_{6583}}{\mathrm{H} \alpha}, \log \frac{[\mathrm{SII}]}{\mathrm{H} \alpha}=\log \frac{[\mathrm{SII}]_{6716+6731}}{\mathrm{H} \alpha},[\mathrm{S} \mathrm{III}]_{1}=[\mathrm{S} \mathrm{III}]_{6716},[\mathrm{~S} \mathrm{III}]_{2}=[\mathrm{S} \mathrm{III}]_{6731}$.

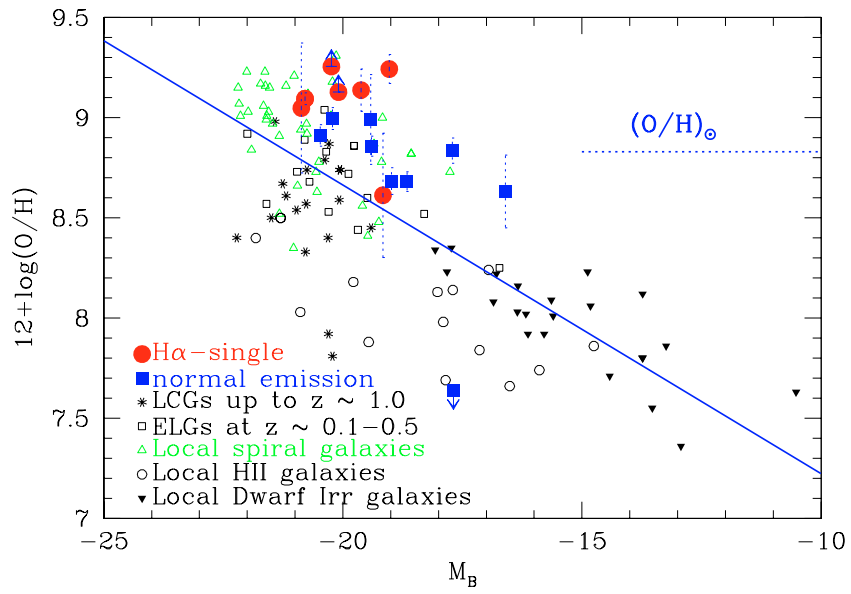

Fig. 9. Oxygen abundances vs. absolute blue magnitudes for the "CFRS H $\alpha$-single" galaxies (filled circles) and the "CFRS normal emission line" galaxies (filled squares), associated with the Luminous Compact Galaxies (LCGs) up to $z \sim 1.0$ (asterisks, from Gruel 2002 and Hammer et al. 2001), the Emission Line Galaxies (ELGs) at $z \sim 0.1-0.5$ (open squares, from Kobulnicky \& Zaritsky 1999, KZ99), the Local Spiral Galaxies (open triangles, from ZKH), the Local H II Galaxies (open circles, from Telles \& Terlevich 1997) and the Local Dwarf Irregular Galaxies (upside-down filled triangles, from Richer \& McCall 1995). All of them have been done using a spectral resolution comparable to ours. The solid line is a linear least-squares fit to local irregular and spiral galaxies (from KZ99). Solar oxygen abundance is shown by the dotted line.

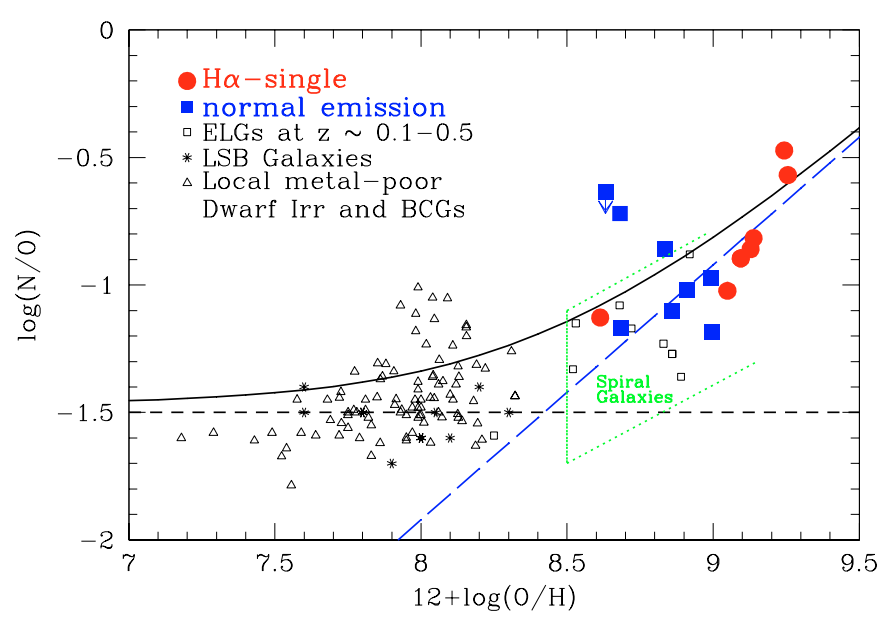

Fig. 10. N/O vs. O/H relations for the "CFRS $\mathrm{H} \alpha$-single" galaxies (filled circles) and the "CFRS normal emission line" galaxies (filled squares), associated with the ELGs at $z \sim 0.1-0.5$ (open squares, from KZ99), the Low Surface Brightness (LSB) Galaxies (asterisks, from van Zee et al. 1997), the Local metal-poor Dwarf Irregular and Blue Compact Galaxies (BCGs) (open triangles, from Kobulnicky \& Skillman 1996 and Izotov \& Thuan 1999). All of them have been done using a spectral resolution comparable to ours. The dotted box for spiral galaxies is taken from KZ99. The horizontal dot-dashed line refers to the primary origin of nitrogen, the sloping dashed line refers to the secondary origin, and the solid line is the combined contribution of a (delayed) primary and a secondary component of nitrogen (taken from Vila-Costas \& Edmunds 1993). 
Table 5. Some derived characteristic parameters of the "CFRS H $\alpha$-single" (the top seven) and the "CFRS normal emission line" (the bottom nine) galaxies; the left part displays the results derived from the higher resolution spectra from the VLT or the CFHT, and the right part displays the results from low resolution CFRS spectra. The subsequent columns in the right part of the table show the measured fluxes of (H $\alpha+[\mathrm{N}$ II]) emission line, the rest-frame $E W$ values of $(\mathrm{H} \alpha+[\mathrm{N} \mathrm{II}])$ emission line (in units of $\AA$ ), the $N_{2}$ parameter, the measured fluxes of $\mathrm{H} \beta$, the aperture correction factor, the $S F R \mathrm{~s}$ with the average extinction $A_{\mathrm{V}}=1\left(S F R_{1}\right)$, the estimated $A_{\mathrm{V}}$ from the CFRS spectra (quoted as $\left.A_{\mathrm{VC}}\right)$ and the derived $S F R \mathrm{~s}$ by using $A_{\mathrm{VC}}\left(S F R_{\mathrm{C}}\right)$. All fluxes of the lines are given in units of $\left(10^{-17} \mathrm{ergs} \mathrm{cm}^{-2} \mathrm{~s}^{-1}\right)$. All $S F R \mathrm{~s}$ are in units of $\left(M_{\odot} \mathrm{yr}^{-1}\right)$.

\begin{tabular}{|c|c|c|c|c|c|c|c|c|c|c|c|c|}
\hline \multirow[b]{3}{*}{ CFRS } & \multirow{2}{*}{$\begin{array}{c}\text { Moderate } \\
\text { VLT }\end{array}$} & \multicolumn{3}{|c|}{ resolution } & \multicolumn{8}{|c|}{ Very low resolution } \\
\hline & & or & CFHT & & & & & CFRS & & & & \\
\hline & $\begin{array}{c}\text { Flux }_{c} \\
(\mathrm{H} \alpha) \\
(2)\end{array}$ & Aper & $\begin{array}{c}L(\mathrm{H} \alpha) \\
\text { ergs s}^{-1} \\
(4)\end{array}$ & $\begin{array}{c}S F R \\
M_{\odot} \mathrm{yr}^{-1} \\
(5)\end{array}$ & $\begin{array}{c}\text { Flux } \\
(\mathrm{H} \alpha+[\mathrm{N} \mathrm{II}]) \\
(6)\end{array}$ & $\begin{array}{l}\text { REW } \\
\text { (7) }\end{array}$ & $\begin{array}{l}N_{2} \\
\text { (8) }\end{array}$ & $\begin{array}{c}\text { Flux } \\
(\mathrm{H} \beta) \\
(9)\end{array}$ & $\begin{array}{l}\text { Aper: } \\
10^{0.4 a} \\
(10)\end{array}$ & $\begin{array}{c}S F R_{1} \\
A_{\mathrm{V}}=1 \\
(11)\end{array}$ & $\begin{array}{l}A_{\mathrm{VC}} \\
\text { (12) }\end{array}$ & $\begin{array}{l}S F R_{\mathrm{C}} \\
\text { (13) }\end{array}$ \\
\hline 03.0364 & $608.9 \pm 100.8$ & 2.14 & $10^{42.26}$ & $31.22 \pm 5.17$ & 94.8 & 44.5 & 0.42 & 0.0 & 2.14 & 7.52 & - & - \\
\hline 03.0365 & $491.3 \pm 102.3$ & 1.92 & $10^{42.04}$ & $16.84 \pm 3.50$ & 121.8 & 52.6 & 0.36 & 0.0 & 1.49 & 5.22 & - & - \\
\hline 03.0578 & $50.1 \pm 20.2$ & 3.39 & $10^{41.11}$ & $3.48 \pm 1.23$ & 18.9 & 46.8 & 0.42 & 0.0 & 2.03 & 1.05 & - & - \\
\hline 03.0641 & $27.7 \pm 8.0$ & 1.51 & $10^{40.96}$ & $1.08 \pm 0.31$ & 25.5 & 20.5 & 0.45 & 0.0 & 1.50 & 1.50 & - & - \\
\hline 03.0711 & $15.2 \pm 1.82$ & 1.37 & $10^{40.70}$ & $0.54 \pm 0.06$ & 14.4 & 32.9 & 0.45 & 0.0 & 1.28 & 0.73 & - & - \\
\hline 03.1014 & $299.6 \pm 97.7$ & 4.58 & $10^{41.73}$ & $19.60 \pm 6.40$ & 103.8 & 24.7 & 0.45 & 0.0 & 1.89 & 4.24 & - & - \\
\hline 22.0717 & $271.2 \pm 85.2$ & 1.15 & $10^{42.01}$ & $9.31 \pm 2.93$ & 19.5 & 10.8 & 0.54 & - & 1.41 & 1.17 & - & - \\
\hline 03.0003 & $21.0 \pm 4.5$ & 3.38 & $10^{40.68}$ & $1.27 \pm 0.27$ & 8.2 & 95.0 & 0.21 & 1.7 & 1.22 & 0.34 & 0.90 & 0.21 \\
\hline 03.0160 & $12.9 \pm 3.3$ & 0.77 & $10^{40.47}$ & $0.18 \pm 0.05$ & 16.9 & 38.9 & 0.45 & 1.2 & 0.77 & 0.35 & 3.13 & 1.99 \\
\hline 03.0149 & $27.7 \pm 4.1$ & 2.87 & $10^{40.92}$ & $1.90 \pm 0.28$ & 17.8 & 51.0 & 0.36 & 3.2 & 2.56 & 1.76 & 0.91 & 1.67 \\
\hline 03.1299 & $1442.1 \pm 281.0$ & 2.28 & $10^{42.31}$ & $37.18 \pm 7.24$ & 224.0 & 77.0 & 0.36 & 16.1 & 2.28 & 9.33 & 3.25 & 58.2 \\
\hline 03.1311 & $531.2 \pm 88.0$ & 1.77 & $10^{41.88}$ & $10.68 \pm 1.77$ & 69.5 & 41.6 & 0.45 & 2.7 & 1.77 & 2.12 & 4.66 & 41.0 \\
\hline 14.1103 & $133.9 \pm 3.0$ & 2.08 & $10^{41.44}$ & $4.50 \pm 0.10$ & 97.7 & 2434.0 & 0.15 & 39.0 & 2.08 & - & 0.00 & 2.86 \\
\hline 14.1117 & $16.0 \pm 2.8$ & 4.73 & $10^{40.44}$ & $1.03 \pm 0.18$ & 19.6 & 69.0 & 0.36 & 4.7 & 4.73 & 2.04 & 0.18 & 1.07 \\
\hline 22.0474 & $102.7 \pm 10.9$ & 1.10 & $10^{41.59}$ & $3.40 \pm 0.36$ & 53.5 & 312.0 & 0.15 & 10.4 & 1.54 & 4.77 & 1.14 & 5.40 \\
\hline 22.1084 & $89.8 \pm 20.8$ & 1.73 & $10^{41.57}$ & $5.15 \pm 1.32$ & 31.5 & 37.7 & 0.45 & 2.3 & 1.73 & 2.74 & 3.05 & 14.5 \\
\hline
\end{tabular}

\subsection{Comparing the SFRs with those from low-resolution CFRS spectra}

To understand more the effect of spectral resolution on the derived SFRs, we also estimated the SFRs of these galaxies from their low-resolution CFRS spectra. For the latter estimates, we have followed the method suggested by TM98 and used the extinction law from Osterbrock (1989), then the dereddened and aperture corrected $\mathrm{H} \alpha$ fluxes are estimated by:

$f(\mathrm{H} \alpha)=\frac{f(\mathrm{H} \alpha+[\mathrm{N} \mathrm{II}])}{1+N_{2}} 10^{c(1.13-0.37)} 10^{0.4 a}$,

where $c=A_{\mathrm{V}} \times 1.47 / 3.2$ (see Sect. 4). For the seven "CFRS $\mathrm{H} \alpha$-single" galaxies, extinction cannot be estimated from the Balmer decrement due to the absence of $\mathrm{H} \beta$ emission; we use the average $A_{\mathrm{V}}=1$, corresponding to $c=$ 0.45 , following TM98. $N_{2}$ reflects [N II] $\lambda 6583$ emission mixed with $\mathrm{H} \alpha$ in the CFRS spectra. We obtained $N_{2}$ values from Fig. $3 b$ of TM98 by considering the rest-frame $E W(\mathrm{H} \alpha+[\mathrm{N} \mathrm{II}])$. $a$ refers to the aperture correction factor by comparing the photometric and spectral magnitudes in $V_{\mathrm{AB}}$ band. Corresponding values are given in the right part of Table 5 (Cols. (6)-(13)).

Figure 11 shows how misleading the SFR estimates are based on low resolution spectra by comparing the corresponding $S_{\text {FRFRS }}$ with the SFR $\mathrm{VLT}_{\mathrm{C} / \mathrm{CFHT}}$ from the moderate resolution spectra. Thus, it may be that TM98 systematically underestimated the SFRs of the "CFRS H $\alpha$-single" galaxies simply because they underestimated the actual extinction coefficients of these galaxies. Conversely, the SFRs of "CFRS normal emission line" galaxies are often overestimated since the underlying absorption beneath $\mathrm{H} \beta$ could not be properly accounted for in low resolution spectroscopy and leads to a severe overestimation of the extinction coefficient properties. This effect probably generates the derived $A_{\mathrm{V}}$ values exceeding 3 or 4 (see Table 5, also Tresse et al. 1996). SFRs of individual galaxies can be only recovered by a proper analysis of the higher quality spectra ( $\left.\mathrm{SFR}_{\mathrm{VLT} / \mathrm{CFHT}}\right)$ at moderate spectral resolution. 
Table 6. The comparison of the total SFRs (in units of $M_{\odot} \mathrm{yr}^{-1}$ ) for the CFRS low- $z$ sample. The adopted interstellar extinction law assumes $(f(\mathrm{H} \alpha)-f(\mathrm{H} \beta)=-0.323)$ and the total galaxies are 110 here by following TM98.

\begin{tabular}{ccc}
\hline \hline & Total $S F R_{\mathrm{VLT} / \mathrm{CFHT}}$ & Total $S F R_{\mathrm{CFRS}}$ \\
\hline 57 “CFRS H $\alpha$-single” galaxies & 696.5 & 160.3 \\
53 “CFRS normal” galaxies & 389.7 & 780.2 \\
Total 110 & 1086.2 & 940.5 \\
\hline
\end{tabular}

Table 7. The comparison of the SFRs (in units of $M_{\odot} \mathrm{yr}^{-1}$ ) of the 16 CFRS low- $z$ galaxies from different extinctions.

\begin{tabular}{ccc}
\hline \hline & $\begin{array}{c}\text { Total } S F R_{\mathrm{VLT} / \mathrm{CFHT}} \\
\text { with well determined } A_{\mathrm{V}}\end{array}$ & $\begin{array}{c}\text { Total } S F R_{\mathrm{VLT} / \mathrm{CFHT}} \\
\text { assuming } A_{\mathrm{H} \alpha}=1\end{array}$ \\
\hline Seven "CFRS H $\alpha$-single" galaxies & 82.1 & 31.4 \\
Nine "CFRS normal" galaxies & 65.3 & 43.4 \\
Total 16 & 147.4 & 74.8 \\
\hline
\end{tabular}

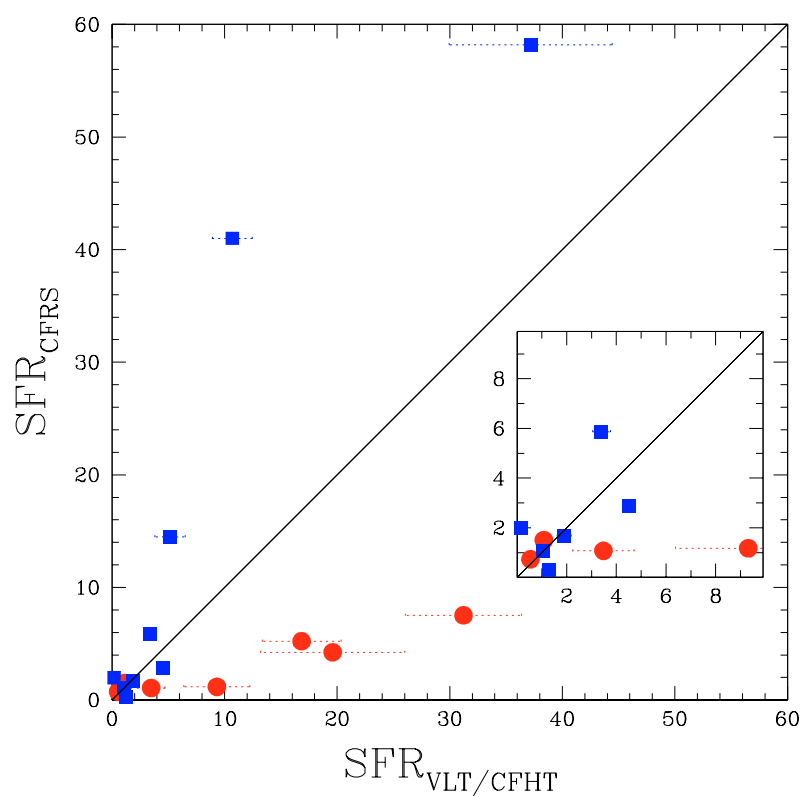

Fig. 11. Comparison between the SFRs of the sample galaxies obtained from the low-resolution CFRS spectra ( $\left.\mathrm{SFR}_{\mathrm{CFRS}}\right)$ and the higher quality spectra $\left(\mathrm{SFR}_{\mathrm{VLT} / \mathrm{CFHT}}\right)$ studied in this work. The small figure in this figure shows the detail around the origin. For $\mathrm{SFR}_{\mathrm{CFRS}}, \mathrm{SFR}_{1}$ were used for the seven "CFRS $\mathrm{H} \alpha$-single" galaxies, and $\mathrm{SFR}_{\mathrm{C}}$ were used for the nine "CFRS normal emission line" galaxies (see Table 5). Symbols as in Fig. 1.

\subsection{Estimates of Cosmic Star Formation Density (CSFD)}

From the above, some qualitative arguments can be used to test the validity of previous works based on low resolution spectroscopy or narrow band filter imagery. Although the latter cannot provide quantitative SFR measurements of individual galaxies, the SFR overestimates and underestimates are almost balanced in the TM98 study (Fig. 11). Table 6 reports the total SFR budget assuming that the seven "CFRS H $\alpha$-single" galaxies and the nine "CFRS normal emission line" galaxies are representative of the whole CFRS sample at low redshift. The difference between the two estimates is only $13 \%$ which is far below the error bars in TM98. Table 5 shows the extinction coefficients of "CFRS normal emission line" galaxies could be much overestimated from the very low resolution spectroscopy. If unrealistic values of $A_{\mathrm{V}}(\geq 3.5)$ are taken to calculate the SFRs of these galaxies, hence the SF density, this could lead to severe overestimations. Recall that for the luminous infrared galaxies, Flores et al. (2004a) never find $A_{\mathrm{V}}$ values larger than 3.5. And the average color excesses, $E(B-V)$, of luminous infrared galaxies (LIGs) studied by Veilleux et al. (1995) are only 0.72, 0.99 and 1.14 in Seyfert 2, H II LIGs and LINERs, respectively.

Fujita et al. (2003) have corrected their $\mathrm{H} \alpha$ luminosities from narrow band filter imagery using $A_{\mathrm{H} \alpha}=1$ which roughly corresponds to $A_{\mathrm{V}} \sim 1.25$. This value is in agreement with our median value for the 16 galaxies studied here. However this correction is related to the power law of the extinction coefficient, leading to important effects related to the large extinction coefficients. Table 7 compares the effect of applying the Fujita et al. (2003) correction to the 16 galaxies studied here. The result is that Fujita et al. (2003) might have underestimated their SF density by a factor close to 2 .

It is out of the scope of this paper to provide a quantitative estimate of the CSFD, because of the small number of objects studied. Indeed, the study here would not help in reconciling the different estimates at low redshift. It however strongly calls for a systematic survey at moderate resolution of a complete sample of galaxies detected from deep narrow band imagery, to correct the $\mathrm{H} \alpha$ luminosities by properly estimating the extinction coefficients.

\section{Discussion and conclusion}

Using moderately high resolution $(R>600)$ and high $\mathrm{S} / \mathrm{N}$ spectra obtained from the VLT and the CFHT, we have studied the properties of a sample of 16 CFRS low redshift galaxies. This sample could be split into seven "CFRS H $\alpha$-single" emission galaxies, and nine "CFRS normal emission line" galaxies, from their spectral properties at the CFRS very low spectral 


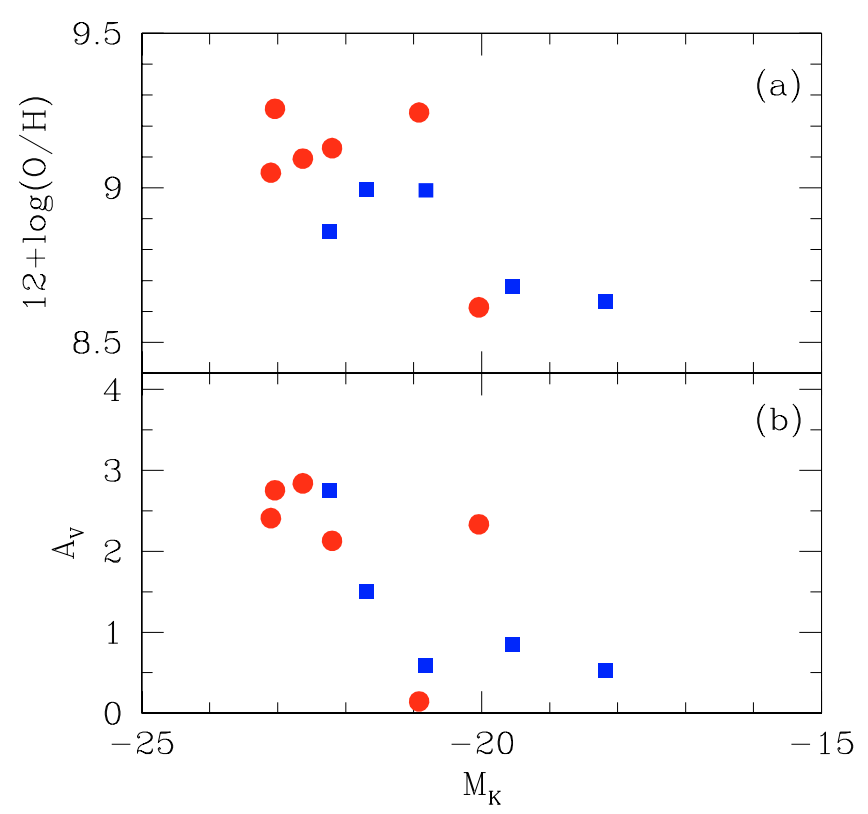

Fig. 12. Oxygen abundances (top) and extinction coefficients (bottom) against absolute $K$ band magnitudes for the sample galaxies. Both relations show the trends that the higher abundances and extinctions are in the more massive stellar systems. Symbols as in Fig. 1.

resolution. Selected from the CFRS sample, these can be taken as representative of the $\mathrm{H} \alpha$-emission field galaxy population at $z \leq 0.3$.

Using the Balmer decrement method $(\mathrm{H} \alpha$ to $\mathrm{H} \beta$ ), we have been able to calculate their interstellar extinction values by properly accounting for the underlying stellar absorption.

Two diagnostic diagrams $(\log ([\mathrm{O} I I I] \quad \lambda 5007 / \mathrm{H} \beta)$ vs. $\log ([\mathrm{N} \mathrm{II}] \lambda 6583 / \mathrm{H} \alpha)$ and $\log ([\mathrm{O}$ III $] \lambda 5007 / \mathrm{H} \beta) \quad$ vs. $\log ([\mathrm{S} \mathrm{II}] \lambda 6716+\lambda 6731 / \mathrm{H} \alpha)$ have been obtained to derive a firm conclusion about the nature of the emission line activity, especially because [N II] emission lines are divided from $\mathrm{H} \alpha$ emission in these higher resolution spectra. Derivation of extinction properties have allowed us to accurately estimate the oxygen and nitrogen abundances, as well as to calculate their SFRs using the extinction-corrected $\mathrm{H} \alpha$ luminosities.

We find that the spectral properties of galaxies at very low spectral resolution can be well understood by the properties of their interstellar media. "CFRS $\mathrm{H} \alpha$-single emission" galaxies shows systematically larger extinction coefficients, and higher oxygen and nitrogen abundances than the rest of the sample. These properties suffice to explain why $\mathrm{H} \beta$ and [O III] $\lambda 5007$ emissions are undetected by low resolution spectroscopy. They can be considered to be mature and massive spirals that lie at low redshifts as can be derived from their $K$ absolute magnitudes (Fig. 12).

SFRs of these low redshift galaxies range from $0.5 M_{\odot} \mathrm{yr}^{-1}$ (Milky Way value) to $40 M_{\odot} \mathrm{yr}^{-1}$ (strong starburst). We also find that the SFRs of individual galaxies cannot be properly derived using low resolution spectroscopy. Indeed, extinction corrections are often large and require a proper account of the underlying stellar absorption to the Balmer lines, which is simply impossible at spectral resolution lower than 600. Previous studies of SF density at low redshifts have assumed average properties for underlying absorption or even for extinction of Balmer line fluxes derived from low resolution spectroscopy. Indeed, the $\mathrm{H} \beta$ line is affected by underlying absorption and extinction in such a complex way that only moderate resolution observations can estimate properly the $\mathrm{H} \alpha / \mathrm{H} \beta$ ratio. Hence, the previous studies may have systematically underestimated the contribution of "CFRS H $\alpha$-single" emission galaxies (the mature and massive systems) and overestimated the contribution of other normal emission line galaxies.

From the data shown here, one can only speculate about the consequences at higher redshifts. Deep surveys preferentially select luminous galaxies in their highest redshift bins, which generally show relatively large extinction coefficients (see Fig. 12). This effect may explain most of the $\sim 60 \%$ gap between the SF density derived by Tresse et al. (2002) (from $\mathrm{H} \alpha$ luminosity, assuming $A_{\mathrm{V}}=1$ ) and that of Flores et al. (1999) (from a combination of IR and UV measurements). Indeed, in a forthcoming paper, Flores et al. (2004b, in preparation) find that one third of the Tresse et al. sample are luminous infrared galaxies, for which $A_{\mathrm{V}}$ should reach values much larger than 1 .

The present study gives a warning for studies based on low resolution spectroscopy aimed at measuring individual galaxy properties (gas chemical abundances, interstellar extinction, stellar population, ages as well as star formation rates and history), particularly for the metal rich and dusty spiral galaxies. Because this affects a large fraction of the galaxies, deriving cosmological star formation density from low resolution spectroscopic surveys could lead to severe biases.

Acknowledgements. We thank the referee for the valuable suggestions which lead us to improve the study. We thank Dr. Rafael Guzmán, Dominique Proust and Jing-Yao Hu for the useful discussions and help. We also thank Dr. Claude Carignan and Mark Neeser for the valuable suggestions and the help to improve the English.

\section{References}

Crampton, D., Le Fèvre, O., Lilly, S. J., \& Hammer, F. 1995, ApJ, 455, 96 (CFRS V)

Flores, H., Hammer, F., Thuan, T. X., et al. 1999, ApJ, 517, 148

Flores, H., Hammer, F., Elbaz, D., et al. 2004a, A\&A, 415, 885

Flores, H., Hammer, F., et al. 2004b, in preparation

Fujita, S. S., Ajiki, M., Shioya, Y., et al. 2003, ApJ, 586, L115

Giovanelli, R., Haynes, M. P., Salzer, J. J., et al. 1995, AJ, 110, 1059

Grevesse, N., \& Sauval, A. J. 1998, Space Sci. Rev., 85, 161

Gruel, N., Ph.D. Thesis

(http://girafdb.obspm.fr/ lirgsiso)

Gruel, N., Hammer F., Flores, H., et al. 2003, in preparation

Hammer, F., Crampton, D., Le Fèvre, O., et al. 1995, ApJ, 455, 88 (CFRS IV)

Hammer, F., \& Flores, H., Proc. of the meeting held in Lyon, France, May 28-June 1st, 2001, ed. F. Combes, D. Barret, \& F. Thévenin (EDP Sciences, Conference Series), 251, in press

Hammer, F., Gruel, N., Thuan, T. X., et al. 2001, ApJ, 550, 570

Hammer, F., Le Fèvre, O., Lilly, S. J., et al. 1997, ApJ, 481, 49 (CFRS XIV)

Izotov, Y. I., \& Thuan, T. X. 1999, ApJ, 511, 639

Jacoby, G. H., Hunter, D. A., \& Christian, C. A. 1984, ApJS, 56, 257

Kennicutt, R. C., Jr. 1998, ARA\&A, 36, 189 
Kennicutt, R. C., Jr., Tamblyn, P., \& Congdon, C. W. 1994, ApJ, 435, 22

Kent, S. M. 1985, ApJS, 59, 115

Kobulnicky, H. A., Kennicutt, R. C., Jr., \& Pizagno, J. L. 1999, ApJ, 514,544

Kobulnicky, H. A., \& Skillman, E. D. 1996, ApJ, 471, 211

Kobulnicky, H. A., \& Zaritsky, D. 1999, ApJ, 511, 118 (KZ99)

Le Fèvre, O., Crampton, D., Lilly, S. J., et al. 1995, ApJ, 455, 60 (CFRS II)

Lilly, S. J., Le Fèvre, O., Crampton, D., et al. 1995a, ApJ, 455, 50 (CFRS I)

Lilly, S. J., Hammer, H., Le Fèvre, O., et al. 1995b, ApJ, 455, 75 (CFRS III)

Lilly, S. J., Schade, D., Le Fèvre, O., et al. 1998, ApJ, 500, 75

Madau, P., Pozzetti, L., \& Dickinson, M. 1998, ApJ, 498, 106

McCall, M. L., Rybski, P. M., \& Shields, G. A. 1985, ApJS, 57, 1

Osterbrock, D. E. 1989, Astrophysics of Gaseous Nebulae and Active Galactic Nuclei (Mill Valley, California: University Science Books)

Pascual, S., Gallego, J., Aragon-Salamanca, A., et al. 2001, A\&A, 379,798
Richer, M. G., \& McCall, M. L. 1995, ApJ, 445, 642

Salpeter, E. E. 1955, ApJ, 121, 161

Seaton, M. J. 1979, MNRAS, 187, 73

Simard, L., Willmer, C. N. A., Vogt, N. P., et al. 2002, ApJS, 142, 1

Telles, E., \& Terlevich, R. 1997, MNRAS, 286, 183

Thurston, T. R., Edmunds, M. G., \& Henry, R. B. C. 1996, MNRAS, 283, 990

Tresse, L., Hammer, F., Le Fèvre, O., et al. 1993, A\&A, 277, 53

Tresse, L., \& Maddox, S. J. 1998, ApJ, 495, 691 (TM98)

Tresse, L., Maddox, S. J., Le Fèvre, O., \& Cuby, J.-G. 2002, MNRAS, 337, 369

Tresse, L., Rola, C., Hammer, F., et al. 1996, MNRAS, 281, 847 (CFRS XII)

van Zee, L., Haynes, M. P., \& Salzer, J. J. 1997, AJ, 114, 2479

van Zee, L., Salzer, J. J., Haynes, M. P., et al. 1998, AJ, 116, 2805

Vila-Costas, M. B., \& Edmunds, M. G. 1993, MNRAS, 265, 119

Veilleux, S., Kim, D.-C., Sanders, D. B., et al. 1995, ApJS, 98, 171

Veilleux, S., \& Osterbrock, D. E. 1987, ApJS, 63, 195

Zaritsky, D., Kennicutt, R. C., \& Huchra, J. P. 1994, ApJ, 420, 87 $(\mathrm{ZKH})$ 